\title{
Cross-synaptic synchrony and transmission of signal and noise across the mouse retina
}

\author{
William N Grimes ${ }^{1}$, Mrinalini Hoon², Kevin L Briggman³ ${ }^{3}$ Rachel O Wong², \\ Fred Rieke ${ }^{1 *}$
}

1'Department of Physiology and Biophysics, Howard Hughes Medical Institute, University of Washington, Seattle, United States; ${ }^{2}$ Department of Biological Structure, University of Washington, Seattle, United States; ${ }^{3}$ Circuit Dynamics and Connectivity Unit, National Institute of Neurological Disorders and Stroke, Bethesda, United States

*For correspondence: rieke@u. washington.edu

Competing interests: The authors declare that no competing interests exist.

Funding: See page 18

Received: 04 July 2014 Accepted: 26 August 2014 Published: 01 September 2014

Reviewing editor: Ronald L Calabrese, Emory University, United States

(c) This is an open-access article, free of all copyright, and may be freely reproduced, distributed, transmitted, modified, built upon, or otherwise used by anyone for any lawful purpose. The work is made available under the Creative Commons CCO public domain dedication.

\begin{abstract}
Cross-synaptic synchrony-correlations in transmitter release across output synapses of a single neuron-is a key determinant of how signal and noise traverse neural circuits. The anatomical connectivity between rod bipolar and A17 amacrine cells in the mammalian retina, specifically that neighboring A17s often receive input from many of the same rod bipolar cells, provides a rare technical opportunity to measure cross-synaptic synchrony under physiological conditions. This approach reveals that synchronization of rod bipolar cell synapses is near perfect in the dark and decreases with increasing light level. Strong synaptic synchronization in the dark minimizes intrinsic synaptic noise and allows rod bipolar cells to faithfully transmit upstream signal and noise to downstream neurons. Desynchronization in steady light lowers the sensitivity of the rod bipolar output to upstream voltage fluctuations. This work reveals how cross-synaptic synchrony shapes retinal responses to physiological light inputs and, more generally, signaling in complex neural networks.

DOI: 10.7554/eLife.03892.001
\end{abstract}

\section{Introduction}

Everyday activities depend on the reliability of neural computation. Yet the mechanistic building blocks of the underlying circuits can exhibit highly stochastic behavior. Understanding neural computation requires bringing these two perspectives together, in particular, it requires identifying, under physiological conditions, the sources of noise in neural signals and how such noise is controlled (Deneve et al., 2001; Averbeck et al., 2006). Sensory systems, for example, must encode and transmit physiological stimuli accurately and quickly based on inherently stochastic processes such as transduction and synaptic transmission.

Neural circuits share a number of common architectural features. Cross-synaptic synchrony (CSS) synchronization of transmitter release at different output synapses of a single neuron-is an important factor in how these common architectural features will impact the coding and transmission of physiological signals (Salinas and Sejnowski, 2000; Rosenbaum et al., 2013, 2014). For example, the strength of network correlations generated by divergent synaptic output from a single presynaptic neuron depends directly on CSS. Similarly, if CSS is high, multiple parallel synapses between a pre- and postsynaptic neuron can enhance transmission of upstream noise and mitigate the impact of intrinsic synaptic noise. Circuits using graded signals or action potentials share these issues because the small number of vesicles associated with transmission of physiological signals cause synaptic release to vary even for nominally fixed presynaptic signals such as action potentials (Del Castillo and Katz, 1954; Allen and Stevens, 1994). 
elife digest The human eye is capable of detecting a single photon of starlight. This level of sensitivity is made possible by the high sensitivity of photoreceptors called rods. There are around 120 million rods in the retina, and they support vision in levels of light that are too low to activate the photoreceptors called cones that allow us to see in color. This is why we cannot see colors in the dark.

Signals are relayed through the retina via a circuit made up of multiple types of neurons. The activation of rods leads to activation of cells known as 'rod bipolar cells' which, in turn, activate amacrine cells and ganglion cells, with the latter sending signals via the optic nerve to the brain. All of these neurons communicate with one another at junctions called synapses. Activation of a rod bipolar cell, for example, triggers the release of molecules called neurotransmitters: these molecules bind to and activate receptors on the amacrine cells, enabling the signal to be transmitted.

For the brain to detect that a single photon has struck a rod, the eye must transmit information along this chain of neurons in a way that is highly reliable while adding very little noise to the signal. Grimes et al. have now revealed a key step in how this is achieved.

Electrical recordings from the mouse retina revealed that, in the dark, small fluctuations in the activity of rod bipolar cells lead to the near-deterministic release of neurotransmitters. This reduces the impact of random fluctuations in neurotransmitter release produced at individual synapses and ensures that the signals from rod bipolar cells (and thus from rods) are transmitted faithfully through the circuit with minimal added noise. As light levels increase, this tight synchrony of transmitter release breaks down, reducing the sensitivity to individual photons.

Given that many other brain regions share the features that enable retinal cells to coordinate the release of neurotransmitters, this mechanism might be used throughout the brain to increase the signal-to-noise ratio for the transmission of information through neural circuits.

DOI: 10.7554/eLife.03892.002

Two issues have hindered progress in understanding the importance of cross-synaptic synchrony for neural signaling. First, quantitative anatomical information about convergent and divergent wiring patterns is essential for understanding the influence of CSS, but such information is lacking for most brain regions (Denk et al., 2012). Second, the experimental conditions for studying neurotransmitter release biophysically often preclude studying physiological signaling in the same circuit. Retinal signaling at low light levels provides an opportunity to tackle these issues directly because of the wealth of available anatomical information (Kolb, 1970; Kolb and Famiglietti, 1974; Tsukamoto et al., 2001; Tsukamoto and Omi, 2013) and the ability to study synaptic mechanisms in the context of physiological light stimuli (Sampath and Rieke, 2004; Dunn and Rieke, 2008; Oesch and Diamond, 2011).

In starlight, signals from rod photoreceptors traverse the retina through the specialized rod bipolar circuit (for review see Bloomfield and Dacheux, 2001). Behavioral and physiological studies indicate that the sensitivity of this circuit approaches limits set by noise in the rod photoreceptors themselves, indicating that little noise is generated at downstream synapses (for review see Field et al., 2005). Rod bipolar cells (RBCs) provide a key component of this circuit; they receive dendritic input exclusively from rods, and provide output to several types of amacrine cells via anatomically distinct and stereotypic connections (Nelson and Kolb, 1984; Tsukamoto and Omi, 2013). Here, we exploit the distinct anatomical features of these connections to quantitatively characterize the role of cross-synaptic synchrony in retinal signaling at low light levels. We find that RBC output synapses can exhibit nearperfect synchronization for small physiological changes in presynaptic voltage such as those encountered near visual threshold. This reveals a surprisingly low level of stochastic behavior at individual synapses under dark-adapted conditions.

\section{Results}

Our aims were (1) to determine the degree of synchronization of RBC output synapses, (2) to identify the factors that control synchronization, and (3) to measure the impact of synchronization on the transmission of signal and noise through the rod bipolar circuit. 


\section{Same synaptic input, very different signal}

All and A17 amacrine cells provide the two main postsynaptic targets of RBCs. As described below, the connectivity between RBCs and these postsynaptic neurons provides an opportunity (1) to measure cross-synaptic synchrony (CSS) using paired A17 recordings, and (2) to examine its role in neural transmission by recording feedforward signaling in the All amacrine cell and other downstream circuit elements.

Each rod bipolar cell releases glutamate from 50 ribbon synapses (Sterling and Lampson, 1986; Tsukamoto et al., 2001; Tsukamoto and Omi, 2013; Mehta et al., 2014) (Figure 1A). These specialized synapses often exist as dyads, in which each presynaptic ribbon is shared by two postsynaptic targets, most commonly one All (Figure 1A, purple) and one A17 (Figure 1A, green) amacrine cell. All and A17 amacrine cells differ in morphology, wiring configuration and functional contributions to retinal processing. All amacrine cells convey rod-mediated signals to ganglion cells via gap junctions made with the axon terminals of On cone bipolar cells and via glycinergic synapses made with both the axon terminals of Off cone bipolar cells and the dendrites of Off ganglion cells. They have a compact dendritic field $(\sim 40 \mu \mathrm{m})$ and receive direct synaptic input from $\sim 10 \mathrm{RBCs}$. A17 amacrine cells provide GABAergic feedback inhibition to RBC axon terminals. They have a wide dendritic field $(\sim 300 \mu \mathrm{m})$ and collect input from more than 100 RBCs, which provide their only known source of excitation. Central to our work here, each RBC can make tens of synaptic contacts onto a single All amacrine cell (Tsukamoto and Omi, 2013), but typically only one synaptic contact onto a single A17 (Ellias and Stevens, 1980; Nelson and Kolb, 1985; Zhang et al., 2002; Grimes et al., 2010).

Spontaneous excitatory synaptic inputs to voltage-clamped All (Figure 1B) and A17 (Figure 1C) amacrine cells differed dramatically (identical recording conditions, see 'Materials and methods'). Both cell types received substantial excitatory input in complete darkness as evinced by the suppression of holding current and noise by $10 \mu \mathrm{M}$ NBQX, an AMPA receptor antagonist (data not shown); however, spontaneous current fluctuations observed in All amacrines were much larger in amplitude $\left(\sigma^{2}=1000\right.$ $\left.\pm 154 \mathrm{pA}^{2}, \mathrm{n}=20\right)$ than those observed in $\mathrm{A} 17$ amacrines $\left(\sigma^{2}=72 \pm 22 \mathrm{pA}^{2}, \mathrm{n}=17\right.$; Figure 1E).

All amacrine cells receive conventional excitatory (i.e., glutamatergic) synaptic input from RBCs and direct electrical input from other Alls and cone bipolar cells via gap junctions. Deletion of connexin-36 disrupts the gap junctional input ( Gjd2 knockout mouse, note: this mouse is also commonly referred to as the Cx36 knockout mouse; Deans et al., 2001, 2002); under these conditions, All input currents continued to exhibit large variability in darkness (Figure 1D,E). Variability in the All inputs was insensitive to pharmacological block of the receptors mediating feedforward (to the All) and feedback (to the RBC axon terminal) inhibition (Figure 1E). Together, these results indicate that the large spontaneous fluctuations in the All inputs arise from excitatory RBC inputs and do not require synaptic inhibition.

How can synaptic inputs from RBCs to All and A17 amacrine cells differ so markedly? Multiple factors, such as differences in the cells' electrical properties, could contribute; we hypothesized that a key factor was differences in the connectivity of All and A17 amacrine cells with RBCs and synchronization of output synapses within individual RBC axon terminals. Since A17 amacrine cells receive input, on average, from one ribbon-type synapse per RBC, their synaptic input should not be affected by synchronization across ribbons. All amacrine cells, however, receive inputs from multiple synapses per RBC and hence their inputs should be shaped by RBC CSS. In support of this synchronization hypothesis, closer examination of the noisy All input currents in Gjd2 knockout mice revealed that the largest spontaneous current fluctuations were many times larger in amplitude and total charge than the average miniature excitatory postsynaptic current (mEPSC; see 'Materials and methods'; Figure 1D).

The differences in RBC connectivity with All and A17 amacrines and in excitatory inputs to the two postsynaptic amacrine cells (in darkness) suggest that CSS substantially shapes RBC synaptic output. The RBC's CSS cannot be measured under dark-adapted conditions using imaging approaches because even two-photon (i.e., infrared) imaging produces too much rod activation to maintain the retina in a dark-adapted state (Euler et al., 2009). Instead, as described below, we took advantage of the sparse, stereotyped connectivity between RBCs and A17s to characterize the CSS of RBCs under physiological conditions.

\section{Synchrony of RBC output}

Each A17 amacrine cell is contacted by a large fraction of the RBCs within its dendritic field ( $50 \%$ in rabbit, Zhang et al., 2002); therefore, pairs of $A 17 \mathrm{~s}$ with highly overlapping dendrites receive synaptic contact from many of the same RBCs (i.e., RBCs that are common to both A17s; Figure 2A,B). Because 

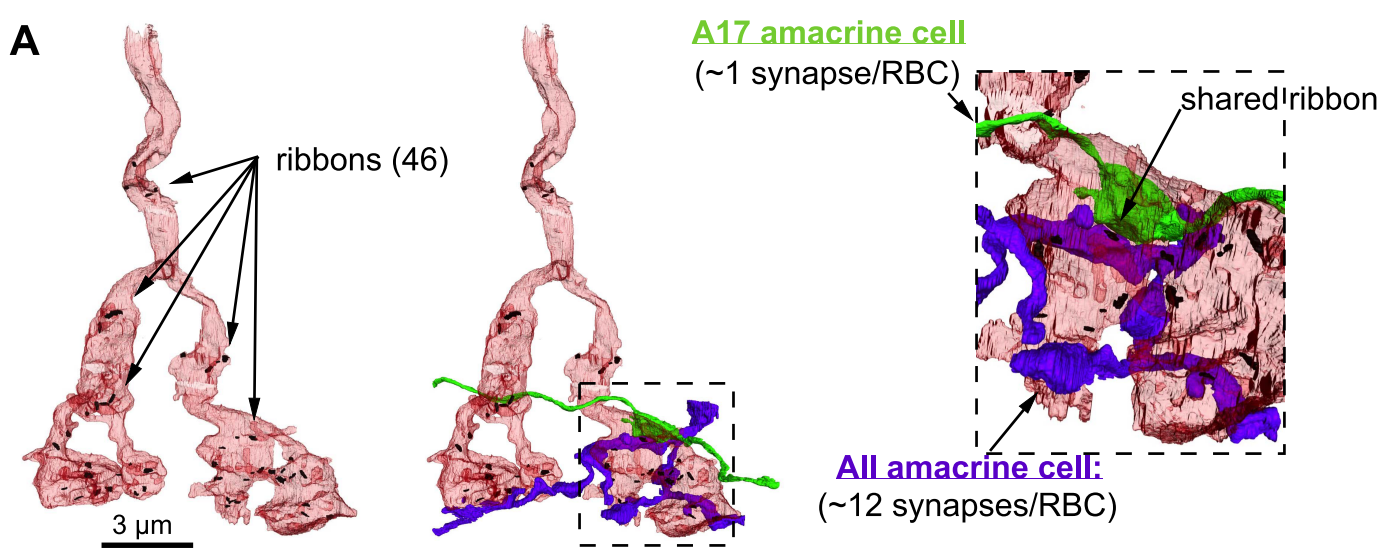

B

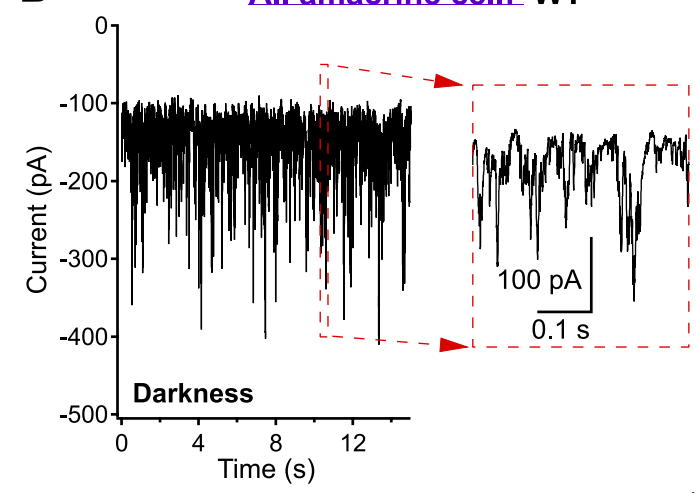

C

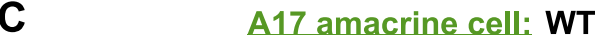

D

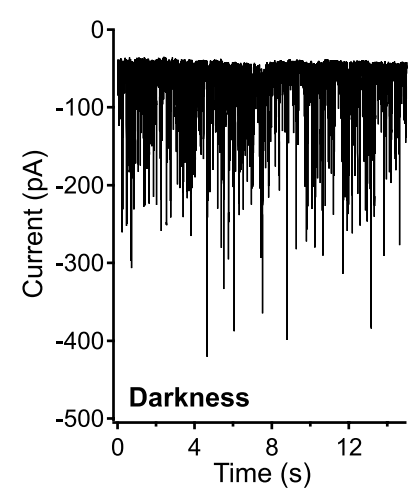

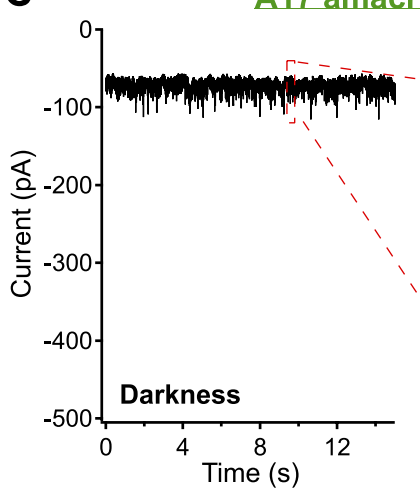

E

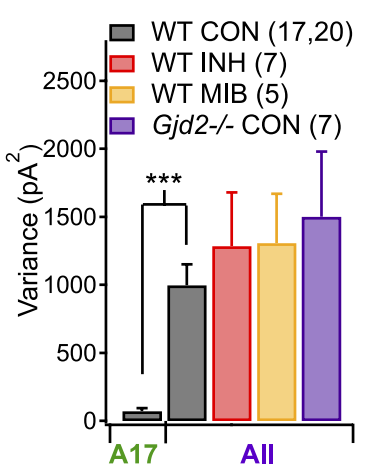

Figure 1. Same presynaptic neuron, very different postsynaptic noise properties. (A) Three-dimensional EM reconstruction of a rod bipolar cell axon terminal (pink), presynaptic ribbons are represented with black markers (46 in total). All (purple) and A17 (green) amacrine cells are complimentary postsynaptic partners at individual RBC ribbon synapses, but unlike the A17, Alls receive synaptic input from multiple presynaptic ribbons. (B and $\mathbf{C}$ ) Voltage-clamp recordings from All (B) and $A 17(\mathbf{C})$ amacrine cells $\left(V_{\text {hold }} \sim-60 \mathrm{mV}\right)$ in retinas from wild-type mice demonstrate that tonic excitatory synaptic input (from RBCs) to these two cell types can be very different under physiological recording conditions. Under dark-adapted conditions large noise events are observed at $\mathrm{RBC} \rightarrow$ All connections $(\mathbf{B})$ but not at $\mathrm{RBC} \rightarrow \mathrm{A} 17$ connections $(\mathbf{C})$. (D) All recordings from retinal slices lacking $\mathrm{Cx36}$-containing gap junctions (i.e., Gjd2 knockout mouse, where electrical synapses between All amacrine cell dendrites and On cone bipolar axon terminals have been eliminated) exhibited similar behavior to recordings from wild-type retina. Under these conditions synaptic events were analyzed. Inset: fast synaptic events (with 10-90\% rise time $\leq 1 \mathrm{~ms}$, i.e., miniature excitatory postsynaptic current or mEPSC) exhibited amplitudes that were less than a tenth of that of the largest events. (E) Population statistics for the synaptic noise recorded from All and A17 amacrine cells in wild type and Gjd2 ${ }^{--}$recordings under dark-adapted conditions (control or drugs). On average, noise recorded from All amacrine cells (WT) was $>10$ times larger than noise recorded from A17 amacrine cells (unpaired $t$ test $\left.p=3 \times 10^{-6}\right)$. Neither an inhibitory cocktail $(2 \mu$ M Strychnine, $20 \mu \mathrm{M}$ SR95531 and $50 \mu \mathrm{M}$ TPMPA) or mibefridil (10 $\mu$ M, T-type $\mathrm{Ca}_{\mathrm{v}}$ channel antagonist) caused a significant change in the noise recorded from All amacrine cells in darkness. DOI: 10.7554/eLife.03892.003 


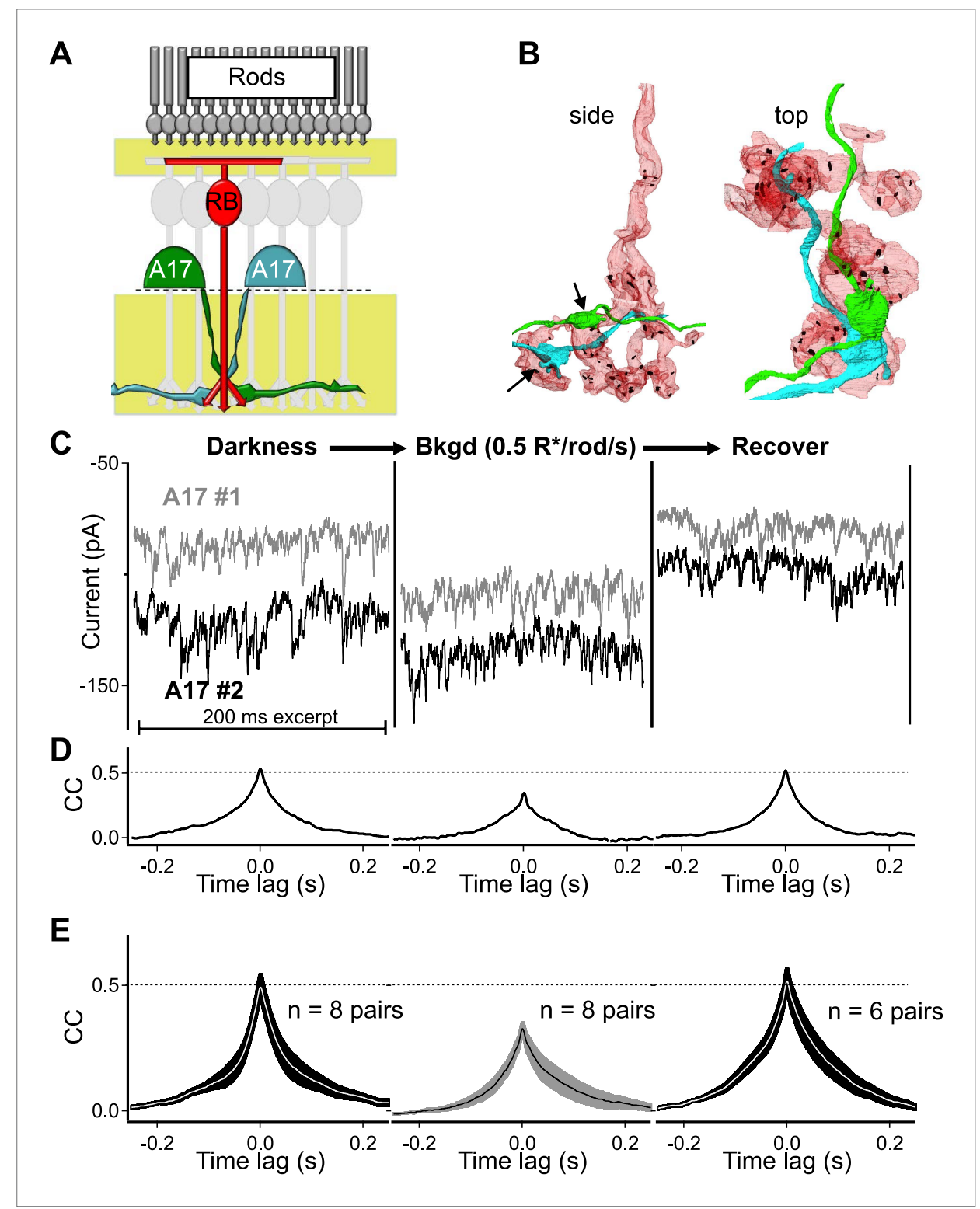

Figure 2. Strong covariation in overlapping A17 amacrine cells reflects highly synchronized cross-synaptic release from individual RBCs under dark-adapted conditions. (A) Paired recordings from neighboring A17 amacrine cells in the wild type retinal slice preparation were used to measure the CSS of RBC output under near-physiological conditions. (B) Pairs of highly overlapping A17 amacrine cells contact many of the same RBCs but at different synaptic locations (arrows). Same RBC serial EM reconstruction as in Figure 1 but with an additional reconstructed A17 amacrine cell dendrite from a different A17 (All is removed). (C-E) Paired recordings from neighboring A17 amacrine cells revealed strong covariation in excitatory synaptic input from RBCs under dark-adapted conditions. Dim backgrounds increased presynaptic release $(\mathbf{C})$ but decreased correlated activity in neighboring A17 amacrine cells ( $\boldsymbol{D}$ and $\mathbf{E} ; \mathrm{p}=0.0053$ for change relative to dark, $\mathrm{n}=8$ pairs). Upon returning to darkness for $\sim 2$ min the strong covariation of presynaptic input recovers to that observed before the background was presented. (E) Population data for cross-correlation functions in darkness (left), $0.5 \mathrm{R} / \mathrm{rod} / \mathrm{s}$ (middle) and after returning to darkness (i.e. recover, right). Thick lines represent the mean, shaded regions represent \pm SEM.

DOI: 10.7554/eLife.03892.004

single ribbons typically provide input to an All-A17 dyad and a single RBC typically contacts an A17 only once (Figure 1A), highly overlapping $A 17$ s receive input from different ribbon-type synapses made by many of the same (i.e., common) RBCs (Figure 2B). Thus synchronized output from synapses within individual RBCs should cause the synaptic input to nearby A17 amacrine cells to covary. 
Paired recordings from neighboring A17 amacrine cells (distance between somas $<80 \mu \mathrm{m}$ ) revealed strong correlations in excitatory synaptic input in the dark (The peak of the cross-correlation function in darkness, that is, the Dark $C_{\text {peak, }}$ was $0.51 \pm 0.03, n=8$ pairs; Figure $2 C-E$ ). Eliminating excitatory synaptic transmission between RBCs and A17s with the AMPA-receptor antagonist NBOX eliminated the correlations (data not shown) and dim backgrounds reversibly reduced correlation strength (dim $\mathrm{CC}_{\text {peak }}=0.34 \pm 0.02, \mathrm{p}=0.0053, \mathrm{n}=8$ pairs; Figure $\left.2 \mathrm{C}-\mathrm{E}\right)$. These results are consistent with strong synchronization of RBC synapses, but they could also reflect electrical coupling between A17 amacrines and/or divergence of upstream rod noise to two or more RBCs.

Direct measurements of electrical coupling between highly overlapping A17s revealed an electrical resistance of $9.8 \pm 1.3 \mathrm{G} \Omega(\mathrm{n}=6$ pairs; Figure $3 \mathrm{~A}, \mathrm{~B})$, more than 30 times the average $A 17$ input resistance $(\sim 300 \mathrm{M} \Omega)$. Substantial dark correlations were present in pairs with the highest resistance $(>15 \mathrm{G} \Omega)$, suggesting at most a small contribution from electrical coupling. We did not attempt to eliminate electrical coupling using genetic manipulations because the connexins forming gap junctions between A17s have not been identified. However, pharmacological experiments described below (see section 'Redundant connections and CSS scale dark noise transmission') provide additional evidence for little contribution of gap junctions to correlations in A17 signals. Contributions from upstream divergence were also minimal, as revealed by direct recordings from pairs of RBCs with touching somas (Figure $3 C_{1} D$ ). Spontaneous excitatory synaptic input to neighboring RBCs was at most weakly correlated in darkness $(0.03 \pm 0.02$, $\mathrm{n}=6$; Figure 3E), indicating minimal correlations in the signals of neighboring RBCs due to rod divergence. Together these experiments indicate that synchronized release from different synapses made by the same RBC dominates the measured correlations in inputs to nearby A17 amacrine cells.
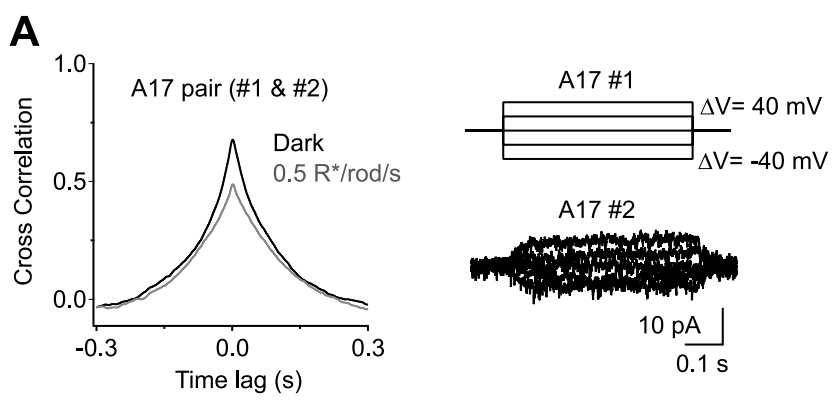

B
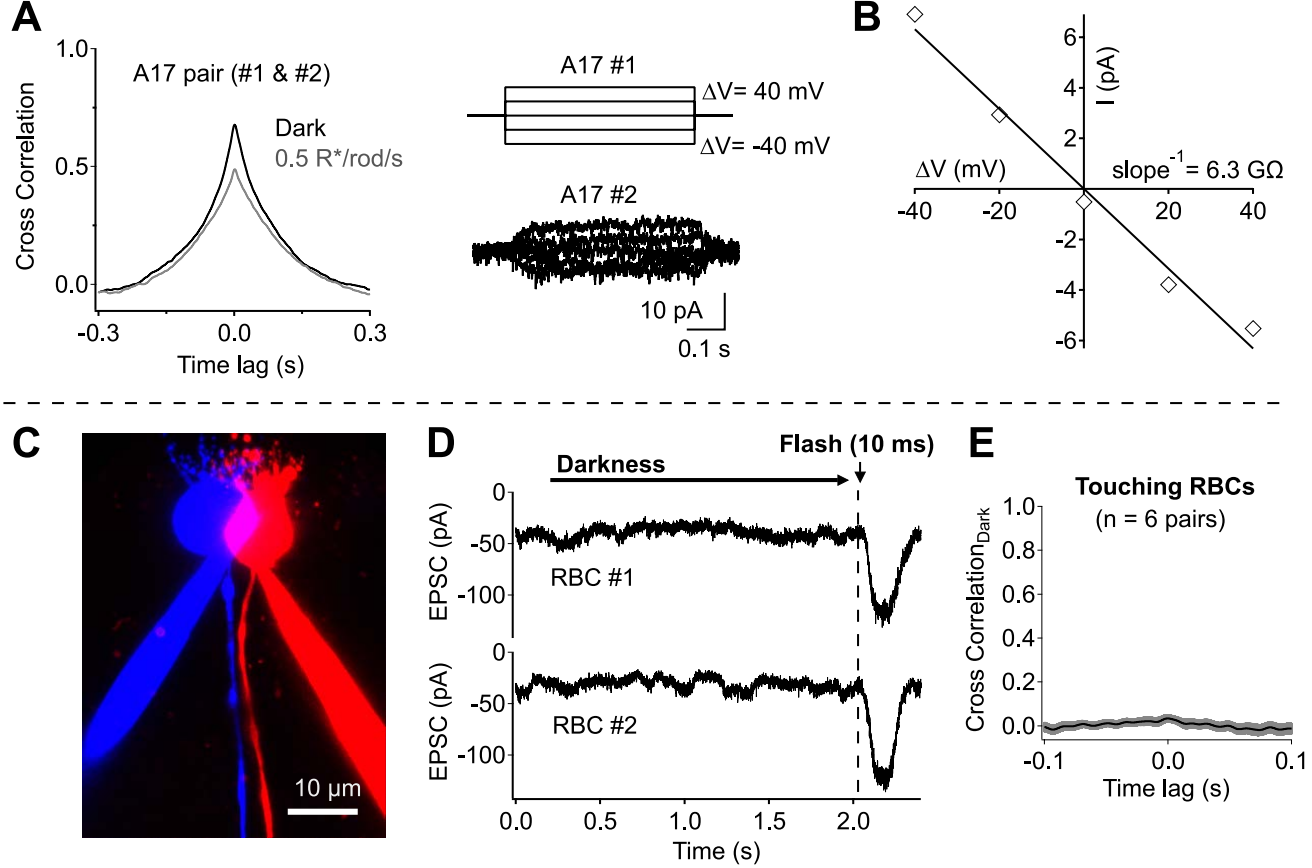

Figure 3. Network divergence and electrical coupling only weakly contribute to correlations observed in highly overlapping A17 amacrine cells. (A and B) Overlapping A17s exhibit weak electrical coupling. (A) Example recording: strong correlations are observed in overlapping A17 amacrine cells (left), even in the absence of significant electrical coupling (right). (B) The gap junctional resistance was estimated by determining the slope of the $\Delta \mathrm{V}$-I relationship. (C-E) Dendritic input to neighboring RBCs is only weakly correlated in darkness. (C) Confocal reconstruction of a paired recording from RBCs with touching somas. (D) Example traces from touching RBC pair. Each recording trial consisted of $2 \mathrm{~s}$ of complete darkness followed by a $10 \mathrm{~ms}$ flash (to monitor sensitivity). (E) Cross correlations were derived for each recording pair before averaging across cells (mean \pm SEM). These experiments were conducted using wild-type retinal slices.

DOI: 10.7554/eLife.03892.005 
The strength of correlations in spontaneous inputs to neighboring A17 amacrine cells in darkness indicated a surprising level of synaptic synchronization considering the lack of visual stimuli and the fact that the presynaptic RBCs are non-spiking neurons. Thus even if the two recorded $A 17 \mathrm{~s}$ receive input from an identical set of RBCs, correlation strengths near 0.5 require that two synapses made by the same RBC must be coactive at least half the time. Thus strong synaptic synchronization requires large, coordinated increases in the probability of release (a notion supported by the electrically compact nature of the RBC, Protti and Llano, 1998) and low intrinsic variability at individual synaptic connections (see 'Discussion'). The remaining experiments investigate the origin and functional impact of such coordinated release.

\section{Quantifying cross-synaptic synchrony}

The strength of correlations in the spontaneous inputs to neighboring A17s will be controlled by the extent to which the cells receive input from common RBCs and by the strength of CSS in the RBC output. As described below, quantitative anatomical measurements of RBC-A17 connectivity (Figure 4) allowed us to relate the measured input correlations to nearby A17 amacrines to the strength of CSS in RBC output. This analysis indicates near-perfect synchronization in the output of RBC synapses in the dark.

Assuming that the measured correlations illustrated in Figure 2 reflect entirely synchronization of RBC synapses, the measured correlation strength (i.e., peak of the cross-correlation function) can be expressed in terms of the RBC's CSS $\left(\beta_{\text {sync }}\right)$, the fraction of RBCs that are common to both A17s (i.e., $P_{\text {sharedi }}$ see 'Materials and methods'), the density of RBC connections, and an electrotonic scaling ( $\gamma$ ) accounting for the attenuation of distal inputs when measured at the soma. Assuming complete dendritic overlap of two neighboring A17 amacrine cells, this relationship can be approximated by summing across circular rings centered on the soma:

$$
C C_{t=0}=\frac{\beta_{\text {sync }} \sum_{r} P_{\text {shared }}(r) n_{r} \exp \left(-2\left(r+r_{0}\right) / \gamma\right)}{\sum_{r} n_{r} \exp \left(-2\left(r+r_{0}\right) / \gamma\right)}
$$

where $r$ is the mean radial distance from the soma for a given ring (Figure 4A,D), $r_{0}$ is the length of the initial descending dendrites (see 'Materials and methods'), and $n_{r}$ is the number of RBCs contacted within each concentric ring (Figure 4F). The numerator in Equation 1 represents the fraction of inputs to one A17 shared with the other, weighted by the electrotonic attenuation and the degree of synchrony. The denominator represents the total input to the cells. The assumptions in the model-for example, complete dendritic overlap of the two cells-cause Equation 1 to underestimate the synchrony required to explain a given correlation strength.

We estimated the anatomical parameters of Equation 1 by filling individual A17 amacrine cells with Lucifer yellow (Figure 4A) and labeling RBCs using antibodies against PKCa (Haverkamp and Wassle, 2000) (see 'Materials and methods'). A17 synaptic inputs were located by the small, bead-like varicosities along their dendrites (Figure 4A). Synaptic contacts between RBCs and A17s were identified by assessing volume overlap in 3-D between the postsynaptic A17 varicosities and the presynaptic PKCalabeled RBCs (Figure 4B, $C$; see 'Materials and methods'). Connectivity was assessed in concentric rings $(\Delta r=20 \mu \mathrm{m})$ centered on the cell body of each $A 17$ and plotted as a function of radial distance (Figure 4E). $\mathrm{P}_{\text {shared }}$ in Equation 1 represents the fraction of the RBC inputs to a given $A 17$ that are shared by an overlapping $A 17$; assuming that the two $A 17 \mathrm{~s}$ are independently wired, $P_{\text {shared }}$ is equivalent to the $A 17 \rightarrow R B C$ connection probability (Figure 4E). Figure $4 F$ plots the number of synaptic contacts made onto a single A17 per ring $\left(n_{r}\right)$. The electronic distance $(\gamma=32.3 \mu \mathrm{m})$ for the A17 dendrites was taken from previous work (Grimes et al., 2010).

Given the parameters measured above, Equation 1 provides a correspondence between the measured strength of correlations in the inputs to neighboring $A 17 \mathrm{~s}$ and the strength of CSS across RBC synapses (purple line in Figure 4G). In other words, Equation 1 allows us to map correlation strength (x-axis in Figure 4G) to CSS strength (y-axis). The slice preparations used for the paired A17 recordings will disrupt the distal dendrites. To correct for this, we reduced $n_{r}$ for rings with a radius exceeding $20 \mu \mathrm{m}$ (see 'Materials and methods'); this correction affected the relation between CSS and correlation strength less than $5 \%$. Because our focus was on using A17s to monitor RBC output, our results are otherwise relatively insensitive to a loss of dendrites in slicing.

Applying Equation 1 to the A17 paired recordings, we estimate that the measured peak crosscorrelation of $0.51 \pm 0.03$ corresponds to a RBC CSS value of $0.80 \pm 0.08$ in darkness. Dim backgrounds 
A

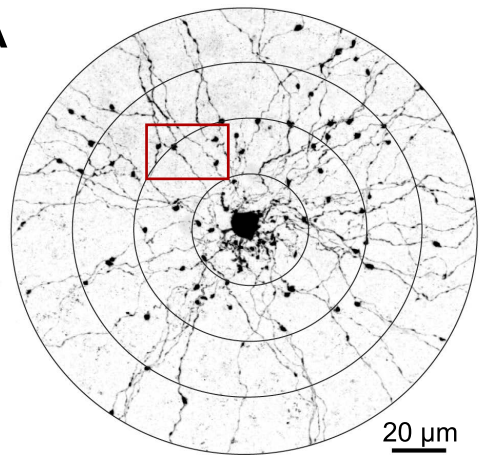

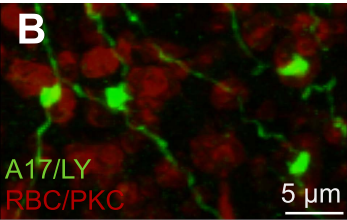
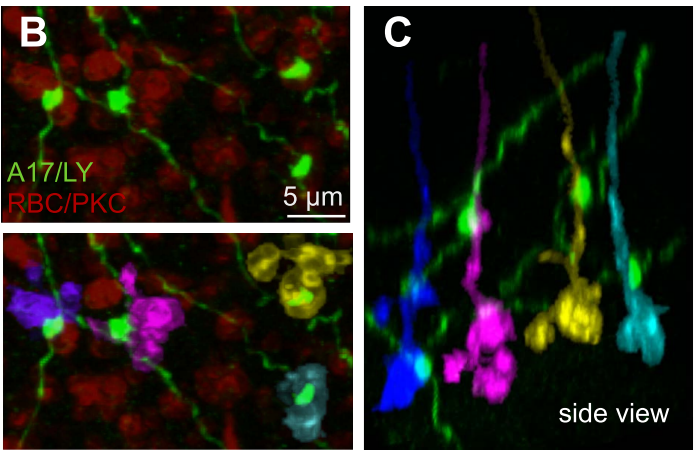

D

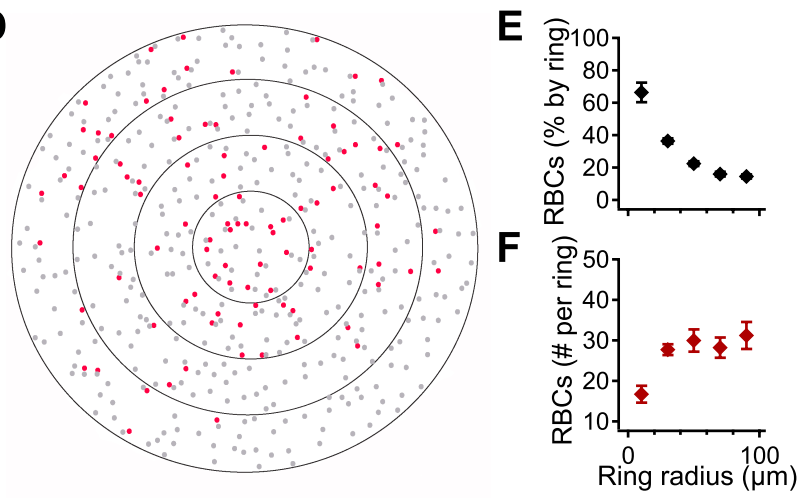

$\mathbf{G}_{1.0}$

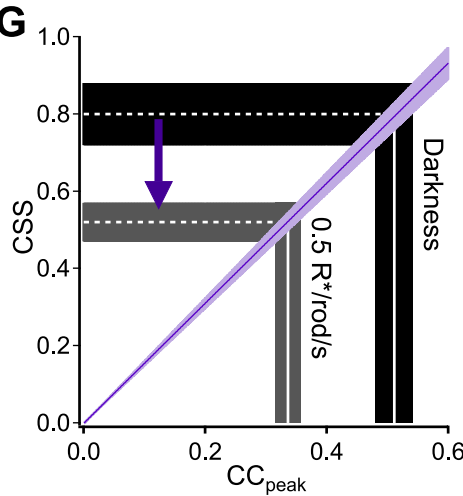

Figure 4. Interpreting A17-A17 correlations in terms of RBC cross-synaptic synchrony. A17 amacrine cell-RBC connectivity was assessed using immunohistochemistry and single-cell injections. (A) A17 amacrine cells have long, thin neurites that are studded by synaptic varicosities. Connectivity between RBCs and Lucifer-yellow (LY) injected A17s were determined within $20 \mu \mathrm{m}$ concentric rings centered on the soma of the injected A17 cell. The outermost ring from panels $\mathbf{A}$ and $\mathbf{D}$ was removed from the image for better viewing of the proximal dendrites but were included in all analyses. (B) Inset from A, RBCs were labeled using antibodies against PKCa (red). Synapses between the RBC and A17 cells were identified by sites of appositions between A17 varicosities and the RBC axon terminal (see 'Materials and methods'). Axonal boutons of four RBCs are colorized separately. (C) Orthogonal rotation of the image stack showing a side view of the four RBC axon terminals connected to the A17 amacrine cell in $\mathbf{A}$ and $\mathbf{B}$. (D) Connectivity map for the A17 cell example in A. Red dots represent the axons of connected RBCs, and gray dots represent the axons of RBCs that did not contact the A17. The percentage $(\mathbf{E})$ and number $(\mathbf{F})$ of RBC connections was determined as a function of radial distance for four injected A17s from four different animals. A17 dendrites traverse $\sim 40 \mu \mathrm{m}$ of the inner plexiform before reaching sublaminas 4 and 5 (where they make the majority of their synaptic contacts with RBCs), therefore, the most central concentric ring actually corresponds to dendritic distances between 40 and $60 \mu \mathrm{m}$, the second ring corresponds to dendritic distances between 60 and $80 \mu \mathrm{m}$ and so on. (G) Changes in the peak amplitude of the cross correlation function reflect luminance-dependent changes in cross-synaptic synchronization as determined by the connectivity and Equation 1 (purple line). Data are presented as mean \pm SEM; SEMs are represented by error bars ( $E$ and $\mathbf{F}$ ) or shaded regions $(\mathbf{G})$. These anatomical experiments were conducted on whole mount retinas taken from Igfbp2-GFP mice. Also see Figure 4-figure supplement 1.

DOI: 10.7554/eLife.03892.009

The following figure supplement is available for figure 4:

Figure supplement 1. Images from Igfbp2-GFP C57BL6 retina.

DOI: 10.7554/eLife.03892.010

reversibly reduced the synchrony of RBC output (CSS: $0.52 \pm 0.05, \mathrm{n}=8$ pairs; Figure $4 \mathrm{G})$. This brings us to two key results. (1) In the dark, release from RBC ribbons is nearly perfectly synchronized. This high CSS, together with differences in connectivity, can at least partially account for the dramatic differences in the properties of RBC synaptic inputs to All and A17 amacrine cells in darkness (Figure 1). (2) This synchronization decreases with steady light. 


\section{Redundant connections and CSS scale dark noise transmission}

What produces a high degree of CSS in the RBC's output in the absence of visual stimuli? First, synaptic failures and other sources of variability at individual synapses must be minimized; a full complement of releasable vesicles and multi-vesicular release likely contribute to minimizing intrinsic synaptic variability at individual RBC synapses (see 'Discussion'). Second, different RBC synapses must experience common fluctuations in release probability so that they become coactive. Fluctuations in the dendritic synaptic input to RBCs from rod photoreceptors and consequent fluctuations in RBC voltage could cause release probability to covary. The pharmacological experiments described below support this proposal.

Rod photoreceptors provide input to RBC dendrites at sign-inverting glutamatergic synapses containing mGluR6 postsynaptic receptors. To reveal the role of upstream rod noise in controlling RBC output, we used agonists and antagonists of these receptors while recording from All and A17 amacrine cells (Figure 5). After collecting dark records from an All or A17 amacrine cell, RBC dendritic input was suppressed by the mGluR6 agonist APB (Slaughter and Miller, 1981). APB increases mGluR6 activity and produces a clear reduction in the mean RBC synaptic input (Sampath and Rieke, 2004). APB substantially decreased both the holding current and noise observed in the inputs to All and A17 amacrine cells, indicating that release from RBCs had been strongly suppressed (Figure 5A-C).

Next, we added the mGluR6 antagonist LY341495. A mixture of receptor agonist and antagonist should suppress RBC voltage fluctuations while permitting control of the mean RBC voltage and release rate via changes in the agonist/antagonist ratio (Ala-Laurila et al., 2011). Indeed, antagonist concentrations could be identified that produced postsynaptic holding currents, and hence RBC synaptic release rates, near those in darkness (dashed lines in Figure 5A,B). Similar agonist/antagonist mixtures maintained the mean RBC voltage while suppressing both light responses and dark noise (Figure 5-figure supplement 1). This manipulation, however, did not restore noise in the inputs to All and A17 amacrine cells to its dark level; the suppression of noise was particularly clear in the All inputs (Figure 5C). Thus similar release rates (dark vs appropriate agonist/antagonist mixture) can produce very different levels of synaptic noise. The larger change in variance of the All inputs compared to the A17 inputs is consistent with the differences in connectivity and a role of CSS in causing small changes in RBC voltage to produce large changes in All input (Figure 1).

The sensitivity of synaptic noise to suppressing fluctuations in RBC dendritic input suggests that RBC voltage fluctuations synchronize synaptic release in the dark. If this is the case, then the strength of correlated synaptic input to neighboring A17s (i.e., CSS) should be sensitive to suppressing RBC voltage fluctuations. Thus, we repeated the pharmacological manipulations of RBC dendritic inputs while recording from $A 17$ pairs (Figure 5D). In control conditions, the peak cross-correlation for these pairs was $0.43 \pm 0.05\left(\mathrm{CSS}_{\text {dark }}=0.71 \pm 0.08, \mathrm{n}=4\right.$ pairs). Suppressing RBC synaptic release with APB eliminated correlations ( $4 \pm 2 \%$ of that in darkness). Restoring the mean release rate with the mGluR6 antagonist modestly increased correlation strength (compared to APB), but it remained significantly less than that in the dark (peak correlation $0.11 \pm 0.03$, corresponding to an estimated CSS of $0.18 \pm 0.05, p=0.0034$, Figure 5D,E).

The agonist/antagonist experiments also provide additional evidence against a primary role for gap junctions between $\mathrm{A} 17 \mathrm{~s}$ in correlating responses of nearby cells. The cross-correlation measures the fraction of the total noise in one cell that is correlated with noise in another cell. Non-rectified electrical connections between two A17s should cause a fraction of the electrical signal of one A17 to be shared with the other. Correlations in the A17 signals produced by gap junctions should hence be insensitive to decreasing the total noise in the A17 signals by suppressing fluctuations in RBC input as the same fraction of noise should remain correlated. The sensitivity of correlation strength to decreasing RBC voltage fluctuations pharmacologically indicates that gap junctions play at most a modest role in producing correlations, confirming the importance of synchrony across RBC synapses.

These results indicate that CSS strength depends on whether noise in the RBC output is produced by upstream sources or is intrinsic to the synapse (Figure 5). Specifically, high CSS in the dark is generated by fluctuations in RBC dendritic input and the resulting fluctuations in RBC voltage.

\section{Light-dependent changes in CSS and noise transmission}

Gain is high within the rod bipolar pathway in the dark (Dunn et al., 2006), helping ensure that signal and noise inherited from the rod photoreceptors rather than noise introduced later in retinal processing 


\section{A All amacrine cell}

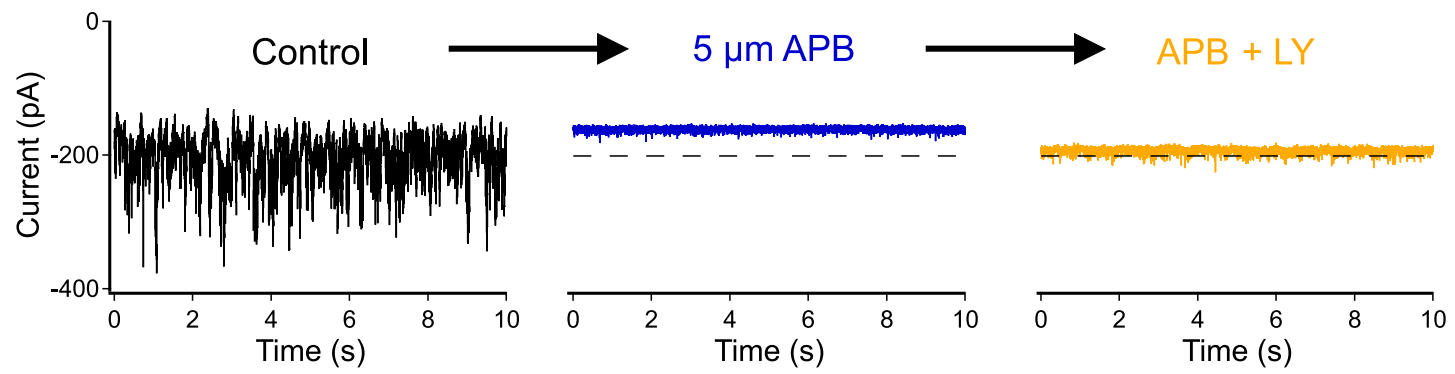

B A17 amacrine cell

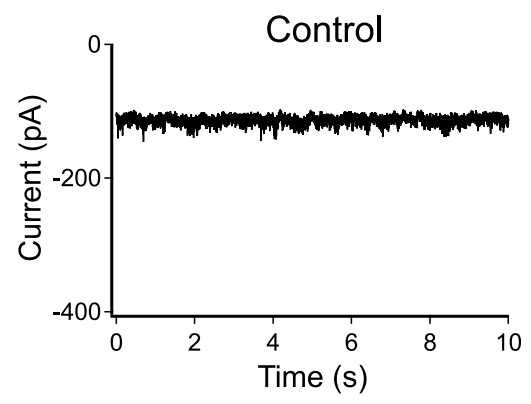

$5 \mu \mathrm{m}$ APB

$A P B+L Y$
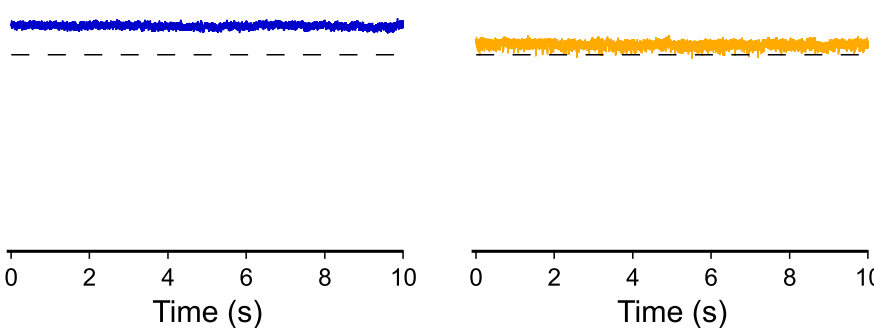

C

D

A17 pairs $(n=4)$
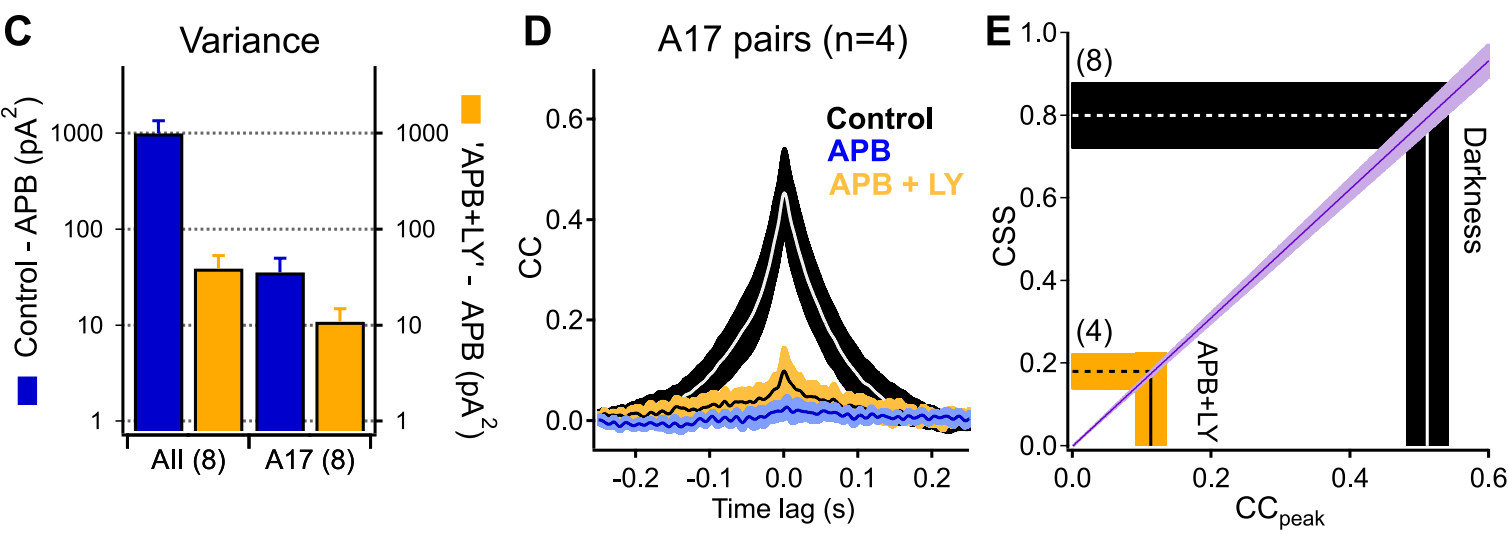

Figure 5. Highly synchronized synaptic noise at RBC $\rightarrow$ All connections under dark-adapted conditions is driven by upstream rod-dependent noise. (A-B) mGluR6 agonists and antagonists can be used to override rod $\rightarrow$ RBC synaptic connections and probe cross-synaptic release properties at RBC $\rightarrow$ All connections. Application of the mGluR6 agonist APB ( $5 \mu \mathrm{M}$, blue-middle) hyperpolarizes the RBCs and shuts down synaptic transmission (i.e., output) to the postsynaptic All (A) and A17 (B) amacrine cells. Addition of the mGluR6 antagonist LY (0.5-2 $\mu \mathrm{M}$, yellow-right) restores tonic release from RBC output synapses (i.e., similar holding current), however, RBC output synapses are now insensitive to fluctuations in transmitter release between rods and RBCs. (C) Summary graph comparing network noise properties observed by All and A17 amacrine cells ( $\mathrm{n}=8$ for all bars). The All amacrine cell inherits (from RBCs) an order of magnitude more network noise than the A17 amacrine cell (blue: $\sigma^{2}{ }_{\text {Con }}-\sigma^{2}{ }_{A P B}$ ) but recovers only a small fraction of this noise when tonic release from RBC synapses is restored (yellow: $\sigma^{2}{ }_{L Y+A P B}-\sigma^{2}{ }_{A P B}$ ). (D) Bath application of 'APB' and 'APB $+L Y^{\prime}$ ' strongly suppress correlated activity in overlapping A17s (APB: $p=0.0088$ for change relative to dark; $A P B+L Y: p=0.0034$ for change relative to dark; $n=4$ pairs). (E) Although the majority of tonic presynaptic glutamate release can be recovered in the presence of 'APB + LY', CSS measurements indicate that RBC output synapses are highly desynchronized under these conditions, thus partially explaining the differences in recovered variance in the All and A17 amacrine cells. Also see Figure 5-figure supplement 1.

DOI: 10.7554/eLife.03892.006

The following figure supplement is available for figure 5 :

Figure supplement 1. Bath application of a solution containing $1 \mu \mathrm{M}$ LY341495 and $5 \mu \mathrm{M}$ APB suppresses dendritic input and voltage fluctuations in RBCs.

DOI: 10.7554/eLife.03892.007 
dominates activity in downstream cells (e.g., All amacrine cells). Redundant connections between RBCs and All amacrine cells and high CSS at these connections provide key elements of such amplification. With increasing light level, the rod bipolar pathway ceases to be the sole route for signals to traverse the retina, as rod and cone signals are conveyed to ganglion cells through the cone bipolar circuits (Xin and Bloomfield, 1999; Deans et al., 2002; Trexler et al., 2005; Manookin et al., 2008). Under these conditions, the need for a high gain pathway is supplanted by a need to suppress noise so as not to contaminate signals in the cone bipolar circuits. Indeed, as described below, we find that noise transmission and CSS decrease with increasing light level.

Transmission of noise from RBCs to All amacrine cells was assessed over a 1000-fold range of light levels ( 0.5-500 R*/rod/s; Figure 6). RBCs depolarize by $\sim 10 \mathrm{mV}$ over this light range, while the mean excitatory synaptic input to All amacrines decreases (Jarsky et al., 2011; Grimes et al., 2014). The variance of the RBC signals changed by less than a factor of two across this luminance range (Figure 6A,D), while the variance of the All amacrine signals decreased 10-fold (Ke et al., 2014) (Figure 6B,D). All noise also decreased in retinas lacking gap junctions between All dendrites and On cone bipolar axons (i.e., Gjd2 knockout mouse; Figure 6D), indicating it was a property of the RBC input to the All.

Steady light changed the kinetics of the noise in the All inputs more than the kinetics of noise in the RBC voltage, as determined from autocorrelograms (Figure 6E). Specifically, the broad temporal correlations characteristic of the All input currents at $0.5 \mathrm{R} * / \mathrm{rod} / \mathrm{s}$ or in the dark were largely absent at $500 \mathrm{R} / \mathrm{rod} / \mathrm{s}$, while the kinetics of the voltage fluctuations in presynaptic RBCs changed relatively little (Figure 6E). The rapid kinetics of noise in the All inputs at $500 \mathrm{R} * / \mathrm{rod} / \mathrm{s}$ matched the kinetics of noise in the presence of the mGluR6 agonist/antagonist mixture introduced in Figure 5 (FWHM of the autocorrelation function was $3.7 \pm 0.3 \mathrm{~ms}$ in steady light vs $4.3 \pm 0.3 \mathrm{~ms}$ in drugs, light $\mathrm{n}=5$, drugs $n=4$; Figure 6F). The broad temporal correlations in the dark are consistent with synchronized release occurring with temporal correlations dictated by the kinetics of RBC voltage fluctuations. The narrow correlations are consistent with release occurring independently of RBC voltage fluctuations. This suggests that the decrease in correlation width produced by the agonist/antagonist mixture or by steady light reflects a relative increase in asynchronous release.

We recorded from A17 pairs to test for a change in CSS with increasing light. Steady light producing $500 \mathrm{R} * \mathrm{rod} / \mathrm{s}$ reduced the peak correlations by more than $\sim 50 \%$ (Figure $6 \mathrm{G}$ ) and reduced the estimated CSS to $0.36 \pm 0.08$ (Figure $6 H$ ). Rod input to the RBC dendrites continued to produce some correlated output even in bright steady light, as indicated by comparison to the lower correlations (and CSS) observed when using the mGluR6 agonist/antagonist mixture (Figure 6H).

The experiments in this section indicate that CSS of the RBC output synapses decreases with increasing light level, such that the synapses becomes less sensitive to fluctuations in RBC voltage. This result is consistent with recent studies showing that the increase in mean RBC voltage with increasing light level produces presynaptic depression by reducing the pool of available vesicles at the RBC $\rightarrow$ All synapse (Dunn and Rieke, 2008; Jarsky et al., 2011; Oesch and Diamond, 2011). Differences in vesicle availability across synapses will increase their variability and thus reduce CSS. Lowered CSS in turn causes less effective transmission of noise or small signals produced upstream in the rod photoreceptors.

\section{Synchronized dark noise in the RB pathway drives correlated activity in retinal ganglion cells}

What impact does the RBC's CSS have on downstream signaling in the retinal output neurons (i.e., retinal ganglion cells, or RGCs)? To answer this question, we recorded synaptic input to pairs of On alpha and Off sustained RGCs (Figure 7). At low light levels, excitatory inputs to On alpha RGCs originate from modulation of the On cone bipolar synaptic output via electrical coupling with All amacrine cells, while inhibitory input to Off sustained RGCs originates directly from glycinergic output of the All (Murphy and Rieke, 2008). Thus the primary source of correlations in these signals comes from fluctuations in All voltage, which, as we show above, is sensitive to CSS.

Excitatory input to the On alpha RGC and inhibitory input to the Off sustained RGC were highly correlated in the dark $\left(C C_{\text {peak }}=0.43 \pm 0.04, \mathrm{n}=5\right.$ pairs; Figure $\left.7 B, C\right)$. Dim steady light $(0.5 \mathrm{R} / \mathrm{rod} / \mathrm{s})$ increased tonic synaptic input to the two cells but, if anything, decreased correlations in synaptic inputs $\left(\mathrm{CC}_{\text {peak }}=0.38 \pm 0.04, \mathrm{n}=5\right.$ pairs; Figure $\left.7 \mathrm{C}\right)$. Bath application of the mGluR6 agonist APB suppressed synaptic input and eliminated correlated activity in On alpha-Off sustained RGC pairs 


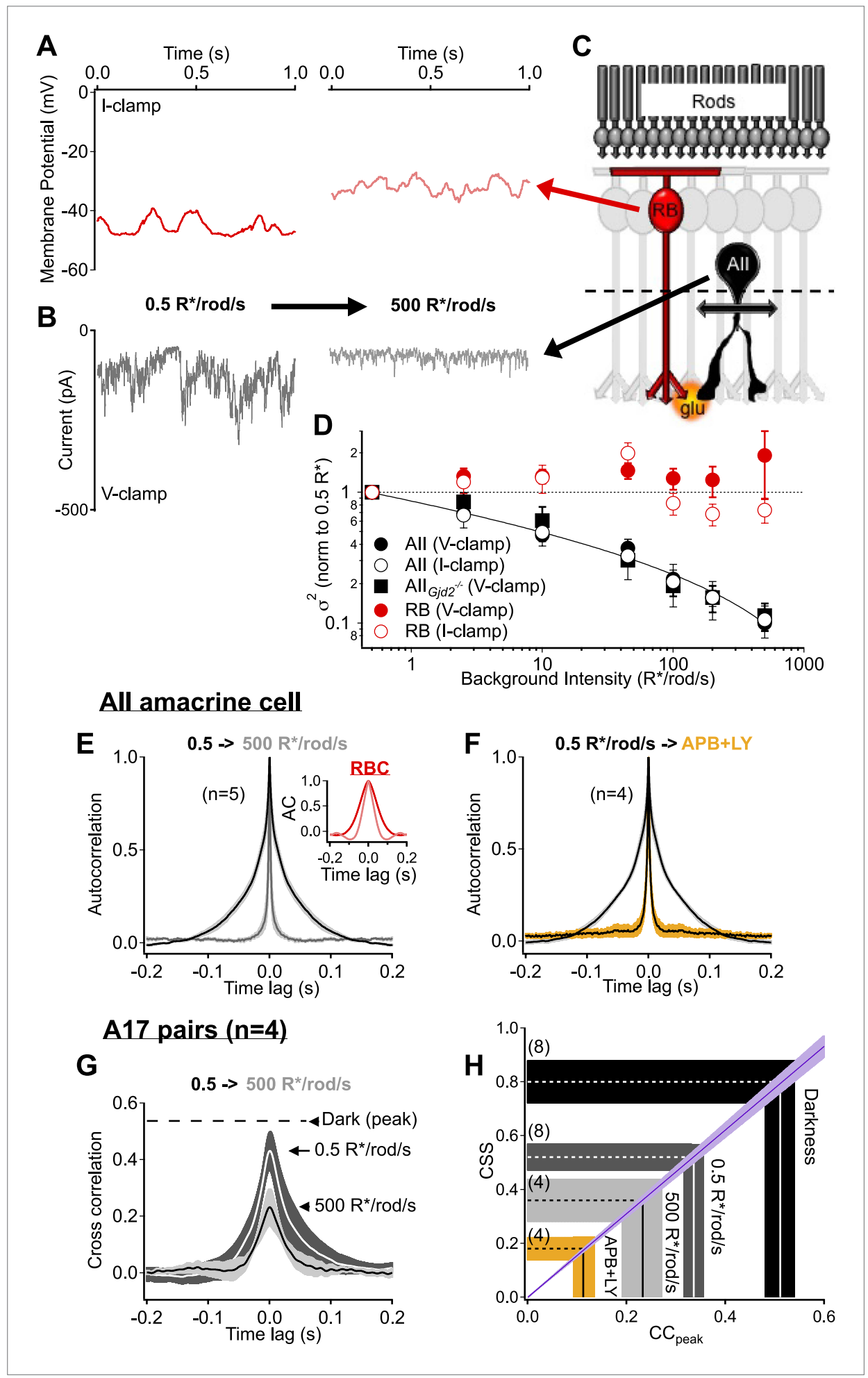

Figure 6. Rod bipolar cell output synapses continue to desynchronize with increasing luminance, reducing the transmission of rod-dependent noise at higher backgrounds. ( $\mathbf{A}-\mathbf{C})$ Individual recordings from a RBC ( $\mathbf{A}$ and $\mathbf{C}$ red) and All amacrine cell (B and $\mathbf{C}$ black) in the presence of a dim background ( $0.5 \mathrm{R} * \mathrm{rod} / \mathrm{s}$, left) and 1000 -fold brighter background (right) illustrate the noise reduction across the RBC. (D) Population data for voltage-clamp recordings of excitatory synaptic input and current clamp recordings of membrane signaling in RBCs (red) and All amacrine cells (black). Noise recorded from RBCs remained relatively constant across this range of backgrounds (comparison Figure 6. Continued on next page 
Figure 6. Continued

of noise at 500 relative to $0.5 \mathrm{R} / \mathrm{rod} / \mathrm{s}, \mathrm{V}$-clamp: $p=0.92, n=7$; I-clamp: $p=0.18, n=4$ ) while noise recorded from All amacrines was reduced $\sim 10$-fold (comparison of noise at 500 relative to $0.5 R^{\star} / \mathrm{rod} / \mathrm{s}, \mathrm{V}$-clamp: $p=0.0039, n=5$; I-clamp: $p=0.019, n=5$ ). All amacrine cell recordings from the retinas of mice lacking connexin36-containing gap junctions (black squares) indicate that neither gap junctions, nor the secondary rod pathway, are required for this transition (comparison of noise at 500 relative to $0.5 R^{*} / \mathrm{rod} / \mathrm{s}, \mathrm{V}$-clamp: $p=0.017, n=5$ ). Error bars represent \pm SEM across cells. (E) Average autocorrelation functions for a population of All amacrine cells recordings under steady-state illumination at 0.5 and $500 \mathrm{R} / \mathrm{rod} / \mathrm{s}$. Slower temporal correlations in the input currents are strongly reduced in the All amacrine cell across this range of luminance. Inset: the reduction in temporal correlations of the RBC voltage response is much less than that observed in the All. (F) The 'LY+APB' manipulation greatly reduces temporal correlations in RBC output, similarly to adaptation to 500 R*/rod/s. (G-H) Paired-recordings from A17 amacrine cells reveal that RBC output synapses become increasingly desynchronized/independent as the retina is adapted to higher luminance $\left(C C_{\text {peak }}=0.23 \pm 0.05\right.$ at $500 \mathrm{R} / \mathrm{rod} / \mathrm{s}$ vs $0.54 \pm 0.06$ in darkness, $n=4$ pairs, $p=0.029$ ). Taken together these data indicate that RBC synapses desynchronize and reduce rod-dependent noise transmission when the retina is adapted to brighter conditions, when the cone-driven circuits are beginning to convey more of the visual information. Thick lines represent means, shaded regions represent \pm SEM. DOI: 10.7554/eLife.03892.011

(Figure 7D). Adding the mGluR6 antagonist LY341495 to restore RBC release to near-dark levels (but with low CSS, see Figure 5) produced weak correlations in synaptic input $\left(C_{\text {peak }}=0.06 \pm 0.05\right.$, $\mathrm{n}=4$ pairs).

Thus, correlations in the RGC synaptic inputs and CSS observed in the RBC synaptic output share a similar dependence on normal dendritic input to RBCs from rod photoreceptors, suggesting that high CSS at the $\mathrm{RBC} \rightarrow$ All synapse plays an important role in creating ganglion cell correlations in darkness. A combination of CSS and redundant connections between RBCs and All amacrine cells help to amplify and transmit small modulations in RBC voltage, whether noise or signals, and by doing so mitigate the impact of noise intrinsic to downstream synapses.

\section{Discussion}

By exploiting the characteristic connectivity between RBCs and two downstream targets, we investigated how cross-synaptic synchrony influences the transmission of physiological inputs through the retina. We found that synchrony across different RBC output synapses was very strong in the dark, such that $\sim 80 \%$ of the vesicle release events occurring at different ribbons within the same RBC axon terminal were synchronous. The high level of cross-synaptic synchrony caused noise in the synaptic inputs to All amacrine cells to be dominated by noise generated in the rod photoreceptors rather than noise generated at the RBC output synapse itself. As light level increased, conditions under which other retinal circuits become active (Manookin et al., 2008), vesicle release at the RBC $\rightarrow$ All synapse transitioned from high CSS to low CSS, thus limiting transmission of upstream signals and noise. We discuss these conclusions and their implications for retinal and neural processing in more detail below.

\section{Implications for photon detection}

The ability of the dark-adapted visual system to detect a small number of absorbed photons (Hecht et al., 1942) places considerable constraints on the underlying mechanisms. For example, behavioral sensitivity requires that rod photoreceptors detect single photons, and we now have a good understanding of how that is achieved mechanistically (reviewed by Rieke and Baylor, 1998). Behavioral sensitivity also requires that retinal synapses maintain low noise so as not to obscure the single photon responses of the rods. Similar requirements on the fidelity of synaptic transmission arise in other neural circuits that sense subtle changes in input.

Synaptic noise first threatens the fidelity of visual signaling at the synapse between rods and RBCs. This is an unusual sign-inverting synapse in which ongoing release of glutamate from the rod photoreceptors acts via metabotropic glutamate receptors to close ion channels in the RBC dendrites (Slaughter and Miller, 1981). Mean release rate at these synapses is highest in the dark, with light exposure leading to a decrease in release rate. In complete darkness, saturation of the postsynaptic metabotropic cascade suppresses the transmission of rod noise; this synaptic saturation enhances the sensitivity of retinal signals more than 10-fold (van Rossum and Smith, 1998; Field and Rieke, 2002; Berntson et al., 2004; Sampath and Rieke, 2004). 
A
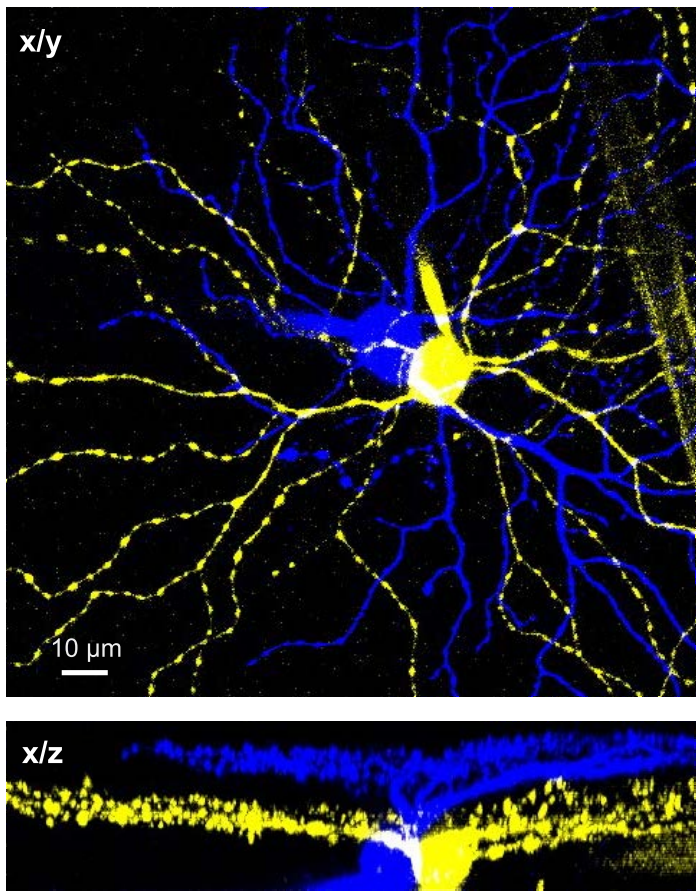

B
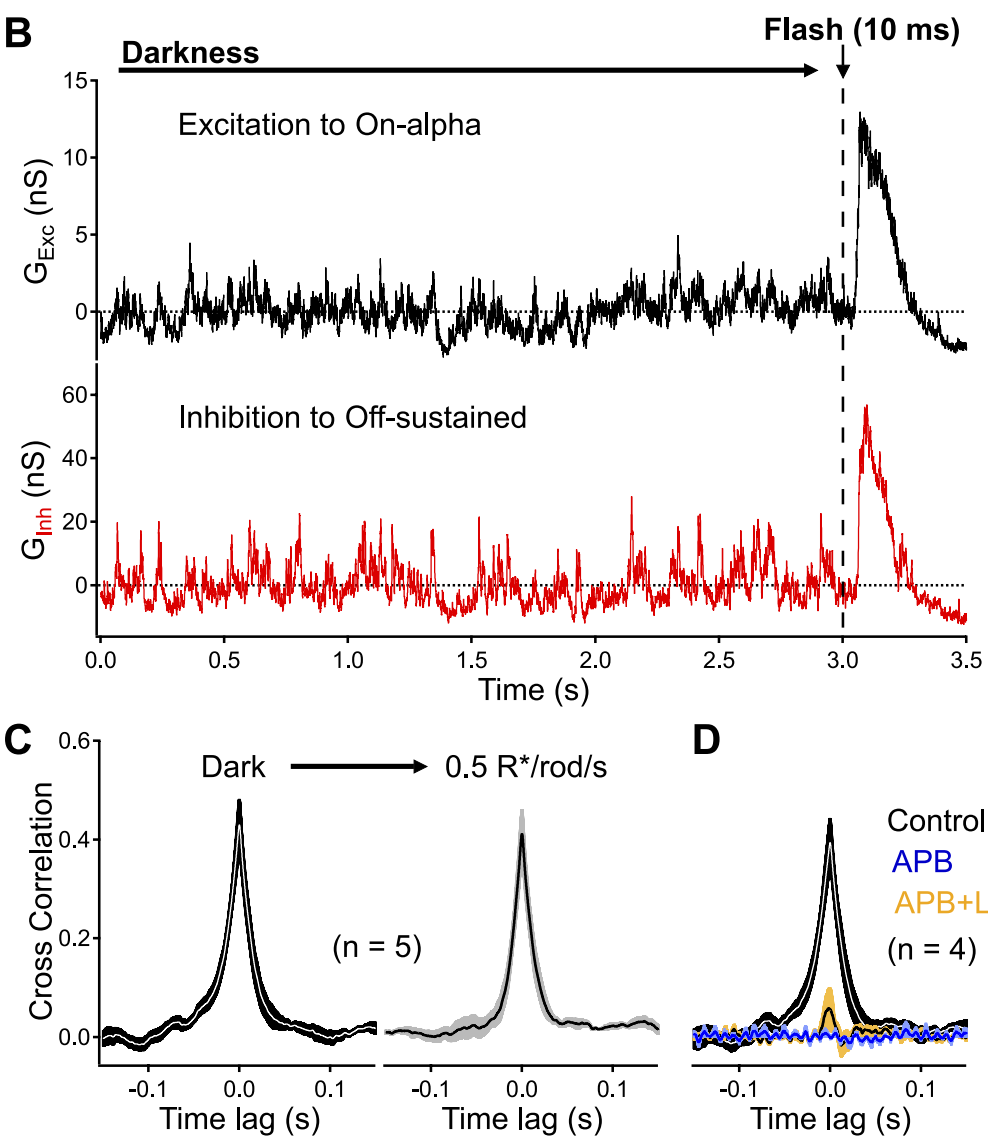

D

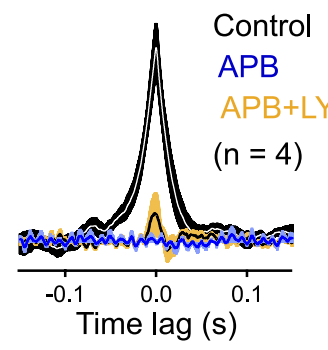

Figure 7. Dark noise and CSS drive strongly-correlated synaptic activity in highly-overlapping On alpha - OFF sustained ganglion cell pairs. (A) Confocal reconstruction of a paired recording from an On alpha (yellow) and OFF sustained (blue) ganglion cell. (B) Example traces of simultaneous recordings of excitatory input to an On alpha (black) and inhibitory input to an Off sustained (red) ganglion cells. At the end of each recording epoch a brief flash was delivered to monitor sensitivity over time. (C) Peak cross correlations measurements from five pairs indicate that synaptic activity is slightly more correlated in the darkness than in the presence of a dim constant background ( $p=0.037$ for a change in $C_{\text {peak }}$ relative to dark, $n=5$ pairs). (D) Suppression of outer retinal activity transmission with APB $(5-10 \mu \mathrm{M})$ eliminates ganglion cell correlations $\left(p=0.0048\right.$ for change in $C C_{\text {peak }}$ relative to dark, $n=4$ pairs). Additional application of LY (at similar concentrations to Figure 5) produces weak correlations in the pairs $\left(p=0.0089\right.$ for a change in $C_{\text {peak }}$ relative to dark, $n=4$ pairs). Thick lines represent means, shaded regions represent \pm SEMs. These experiments were conducted using wild type whole mount retinal preparations.

DOI: 10.7554/eLife.03892.012

The potential pitfalls associated with synaptic noise recur as signals traverse the retina, mainly via conventional chemical synapses. Our work here details how the operation of synapses between RBCs and All amacrine cells maximizes sensitivity to upstream activity while minimizing added noise in darkness. Specifically, highly synchronized vesicle release across parallel RBC output synapses amplifies RBC voltage fluctuations produced by rod signals and noise while minimizing added synaptic noise. Quite surprisingly, achieving the estimated dark CSS of 0.8 requires that the dark fluctuations in RBC voltage produce near-deterministic changes in vesicle release at individual synapses (Figure 8A). Multiple release sites at each ribbon synapse (each RBC ribbon has $\sim 10$ active zones; Figure $8 B$ ), high vesicle availability and postsynaptic receptor saturation (Tong and Jahr, 1994) (Figure 8C) will facilitate low synaptic variability. The low rate of ongoing release in darkness compared to that in the presence of steady light ensures that the readily releasable vesicle pool in the RBC terminal remains full (Singer and Diamond, 2006), an important component of strong synaptic synchronization. These results highlight that the RBC output synapses operate far from a regime in which vesicle release follows Poisson statistics, and this is important for their ability to reliably transmit the small signals forming the basis of night vision. 


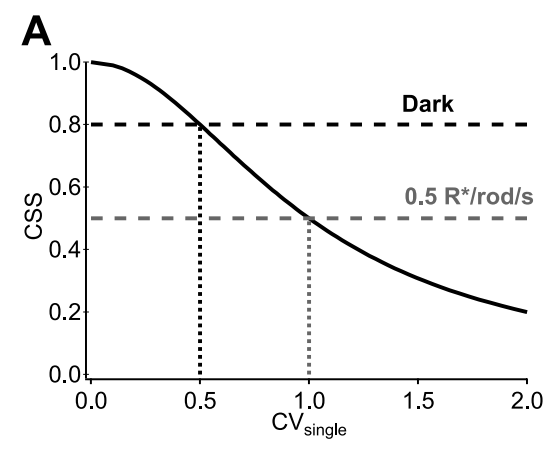

B Single synapse variability, $\mathrm{CV}_{\text {single }}$ (fixed unitary event)

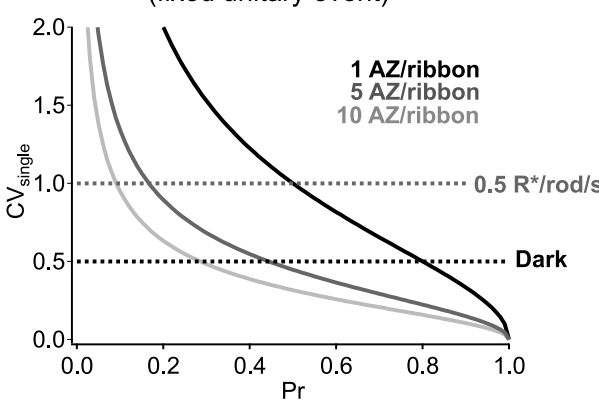

C 10 Independent AZs/Ribbon

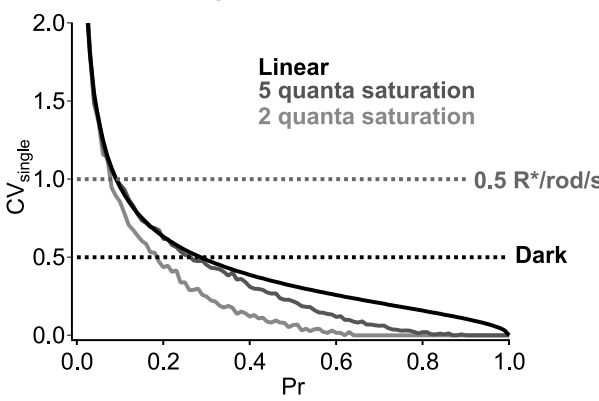

Figure 8. Multiple active zones (AZ) per synapse and low synaptic variability enhance CSS in darkness.

(A) CSS measurements constrain synaptic variability at individual synaptic connections. Assuming a homogeneous release probability, a CSS measurement of 0.8 in darkness indicates that the coefficient of variation (CV) at individual synapses must be $\leq 0.5$. (B) Multiple release sites/active zones improve reliability at individual synapses. Previous work indicates that each RBC ribbon synapse has $\sim 10$ active zones, thus facilitating multivesicular release. (C) Postsynaptic receptor saturation can further improve reliability at individual synapses. If the synaptic receptors are saturated by the release of five or more vesicles (dark gray) then the response to the release of $>5$ vesicles will be identical to the response to five vesicles. This reflects a tradeoff between dynamic range and reliability. DOI: 10.7554/eLife.03892.008 of noise correlations produced by divergence, without morphological changes in the circuit connections.

The control of synaptic output via fluctuations in RBC voltage requires fine control of the synaptic operating point. As described above, if the RBC is too depolarized in the dark the spontaneous release rate will go up (Jarsky et al., 2011), which will both increase stochastic fluctuations in release and deplete the pool of releasable vesicles. If the RBC is too strongly hyperpolarized, the synapse will be ineffective in transmitting single photon responses generated in the rods. Thus effective transmission of single photon responses requires a balance, maintained through the ongoing level of dendritic input to the RBC from the rods and inhibitory feedback onto the RBC synaptic terminal from A17 amacrine cells.

\section{Implications for dynamic interactions between parallel circuits}

Functional and anatomical work shows that most neural circuits contain multiple cell types organized to process circuit inputs in parallel. This architectural similarity highlights several common motifs in neural computation: (1) divergence via multiple output synapses can produce correlated activity in downstream circuit elements (Kazama and Wilson, 2009); (2) integration and processing of functionally dissimilar inputs from different parallel circuits controls computation in many neurons (Olsen et al., 2007; Fischer et al., 2008; Schnell et al., 2010); and (3) neurons and synapses often participate in multiple functional circuits (Munch et al., 2009; Grimes et al., 2014), a possible outcome of the evolutionary pressures to maximize computational capacity while economizing the necessary biological hardware. Cross-synaptic synchrony impacts each of these issues.

Retinal ganglion cells, like output neurons in many neural circuits, exhibit strong noise correlations (Arnett and Spraker, 1981; Mastronarde, 1983; Murphy and Rieke, 2008; Cafaro and Rieke, 2010; Volgyi et al., 2013), and these correlations can be dominated by divergent noise from common upstream circuit elements (Brivanlou et al., 1998; Trong and Rieke, 2008; Ala-Laurila et al., 2011). Cross-synaptic synchrony is a key determinant of whether anatomical divergence will produce correlated signals in downstream neurons. When cross-synaptic synchrony is high, postsynaptic targets of a given neuron will receive highly correlated input. Changes in CSS, such as with mean light level as observed here, will then control the strength 
Changes in CSS could also play an important role in the integration of signals from distinct parallel circuits. For example, as light levels increase from darkness, the need for high gain imposed by detecting sparse photons is reduced and visual perception depends less critically on signals traversing the rod bipolar pathway. Over a substantial range of light levels, visual signals elicit simultaneous activity in rod and cone photoreceptors and their associated circuitry (Naarendorp et al., 2010). These parallel signals are combined through several shared circuit elements (e.g., All amacrine cells) before they are transmitted to targets in the central nervous system. Efficient transmission of noisy rod signals through the rod bipolar pathway could jeopardize cone signals under these conditions. As luminance increases the RBC depolarizes, eventually evoking synaptic depression by way of vesicle depletion and $\mathrm{Ca}_{\mathrm{v}}$ inactivation (Singer and Diamond, 2006; Jarsky et al., 2011; Oesch and Diamond, 2011), mechanisms that likely underlie the decrease in cross-synaptic synchrony we observe here. The observed decrease in synchrony within the rod bipolar pathway serves to decrease transmission of rod noise to shared downstream circuit elements. Other highly interconnected brain regions might use similar mechanisms for dynamically regulating signal transmission in parallel circuits prior to signal integration.

\section{Materials and methods}

\section{Electrophysiology}

Experiments were conducted on whole mount and slice (200 $\mu \mathrm{m}$ thick) preparations taken from dark-adapted Gjd2 knockout (Deans et al., 2001, 2002) or wild-type C57/BL6 mice. Retinas were isolated under infrared visualization and stored in oxygenated $\left(95 \% \mathrm{O}_{2} / 5 \% \mathrm{CO}_{2}\right.$ ) Ames medium (Sigma, St. Louis, MO) at $\sim 32^{\circ} \mathrm{C}$ to $34^{\circ} \mathrm{C}$. Once under the microscope, tissue preparations were perfused by the same Ames solution at a rate of $\sim 8 \mathrm{ml} / \mathrm{min}$. Isolated retinas were either flattened onto polyL-lysine slides (whole mount) or embedded in agarose and sliced as previously described (Dunn et al., 2006; Murphy and Rieke, 2006). Retinal neurons were visualized and targeted for whole-cell recordings using video DIC with an infrared light source (>950 nm). Data in Figures 1-3,5,6 and Figure 5-figure supplement 1 were collected from retinal slices, whereas data in Figures 4,7 and Figure 4-figure supplement 1 were collected from whole mount preparations. To ensure that retinal recordings consistently reflected a dark-adapted state, only one recording (single cell or paired) came from each darkadapted retina preparation (i.e., slice or whole-mount).

Voltage clamp recordings were obtained using pipettes (RGCs: 2-3 M 2 , All and A17 amacrine cells: 5-6 M , bipolar cells: 10-14 M 2 ) filled with an intracellular solution containing (in $\mathrm{mM}$ ): $105 \mathrm{Cs}$ methanesulfonate, 10 TEA-Cl, 20 HEPES, 10 EGTA, 2 QX-314, 5 Mg-ATP, 0.5 Tris-GTP, and 0.1 Alexa (488, 555 , or 750 ) hydrazide ( $280 \mathrm{mOsm}$; $\mathrm{pH} \sim 7.3$ with $\mathrm{CsOH})$. Current clamp recordings used an intracellular solution containing (in $\mathrm{mM}$ ): $123 \mathrm{~K}$-aspartate, $10 \mathrm{KCl}, 10 \mathrm{HEPES}, 1 \mathrm{MgCl}_{2}, 1 \mathrm{CaCl}_{2}, 2 \mathrm{EGTA}$, $4 \mathrm{Mg}$-ATP, 0.5 Tris-GTP, and 0.1 Alexa $(488,555$ or 750) hydrazide ( $280 \mathrm{mOsm} ; \mathrm{pH} \sim 7.2$ with $\mathrm{KOH})$.

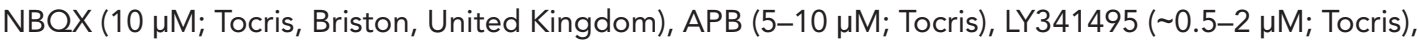
Mibefridil $(10 \mu \mathrm{M})$, or an inhibitory cocktail (20 $\mu \mathrm{M}$ SR95531, $50 \mu \mathrm{M}$ TPMPA and $2 \mu \mathrm{M}$ strychnine; Tocris) was added to the perfusion solution as indicated in Figures 1,5-7 and Figure 5-figure supplement 1 . To isolate excitatory or inhibitory synaptic input, cells were held at the estimated reversal potential for inhibitory or excitatory input of $\sim-60 \mathrm{mV}$ and $\sim+10 \mathrm{mV}$. Absolute voltage values were not corrected for liquid junction potentials $\left(\mathrm{K}^{+}-\right.$based $=-10.8 \mathrm{mV} ; \mathrm{Cs}^{+}-$based $\left.=-8.5 \mathrm{mV}\right)$.

\section{Visual stimuli}

For all experiments, full field illumination (diameter: $560 \mu \mathrm{m}$ ) was delivered to the preparation through a customized condenser from blue (peak power at $470 \mathrm{~nm}$ ) or green (peak power at $510 \mathrm{~nm}$ ) LEDs.

\section{Analysis}

We estimated the strength of CSS in the RBC output based on paired recordings from highly overlapping A17 amacrine cells and the calculation outlined below. The calculation sums over circular disks centered on the soma. The variance in the response of a single cell is then

$$
\sigma_{\text {total }}^{2}=\sum_{r} n_{r} \exp \left(-2\left(r+r_{0}\right) / \gamma\right)
$$

where $n_{r}$ is the number of synaptic contacts and $\gamma$ is the electrotonic scaling factor for synaptic inputs at a particular radial distance (the electrotonic length factor for A17 dendrites comes from Grimes et al., 2010). A17 dendrites traverse $\sim 40 \mu \mathrm{m}$ of the inner plexiform before reaching sublaminas 4 
and 5 (where they make the majority of their synaptic contacts with RBCs); $r_{0}$ accounts for the length of these initial descending dendrites. The common variance can then be defined as

$$
\sigma_{\text {shared }}^{2}=\beta_{\text {sync }} \sum_{r} P_{\text {shared }}(r) n_{r} \exp \left(-2\left(r+r_{0}\right) / \gamma\right)
$$

where $\beta_{\text {sync }}$ is the strength of CSS of RBC synapses (between 0 and 1 ), and $P_{\text {shared }}$ is the percentage of contacted RBCs that are common to the two cells within a particular ring. The cross correlation function at zero time lag is mathematically defined as the shared variance over the geometric mean of the independent variances; in terms of CSS and the A17 paired connectivity the cross correlation function can be defined as in Equation 1.

TaroTools event detection plug-ins (for lgor Pro) were used to examine postsynaptic currents in darkness from All amacrine cells from Gjd2 knockout mice. Miniature excitatory postsynaptic currents were identified by setting the event detection threshold to $-2 \mathrm{pA}$ and requiring a $10-90 \%$ rise time of $1 \mathrm{~ms}$ or less. Fluctuating baselines (likely due to gap junction input) prohibited effective event detection in WT Alls.

All data are presented as mean \pm SEM and two-tailed paired student's $t$ tests were used to test significance unless otherwise noted.

\section{Synaptic modeling}

With the assumption that synapses in the RBC's axon exhibit homogeneous release probability, the cross synaptic synchrony can be related to the variability at individual synapses as

$$
\beta_{\text {sync }}=\frac{1}{1+C V_{\text {single }}^{2}}
$$

where $\mathrm{CV}_{\text {single }}$ is the coefficient of variation for transmission at a single synapse. Variability at individual synapses was modeled explicitly in Figure 8 by examining probabilistic signaling for a varying number of independent release sites/active zones at a given ribbon synapse (with fixed vesicle availability and quantal response). Variability in transmission was calculated assuming no variability in the postsynaptic response to a quantal release event as

$$
C V_{\text {single }}=\frac{\sqrt{1-P r}}{\sqrt{N^{*} \operatorname{Pr}}}
$$

where $N$ is the number of release sites/active zones at a single ribbon synapse. In cases when two or more vesicles are synchronously released at a single synapse, postsynaptic receptors could experience saturation (Tong and Jahr, 1994). This possibility was modeled by equating postsynaptic responses to 2 or more released vesicles. 200 trials were run for each value of $\operatorname{Pr}$ with 10 active zones at a given ribbon. For each trial, a release event was initiated when a randomly generated number was less than Pr. The coefficient of variation was then calculated across trials (SD/mean).

\section{Cell identification}

On alpha-like RGCs, Off sustained RGCs, RBCs, A17 amacrine cells, and All amacrine cells were identified by soma morphology and electrophysiological characteristics. Cell identity was often further confirmed post-recording by imaging the dye-filled arbors (Alexa 488, 555 or 750) using confocal microscopy or epifluorescence.

\section{Light adaptation}

For experiments probing different levels of luminance (Figures 2,6 and 7), we allowed 30-120 s of adaptation at each luminance level before steady state was reached, and data were analyzed.

\section{Cell selection criteria}

Recordings from slice preparations were performed within $\sim 4 \mathrm{hr}$ of retinal dissection, and we specifically targeted neurons that were $\geq 20 \mu \mathrm{m}$ below the surface of the slice. RBCs were selected for when saturating flashes from darkness produced reliable and robust events both before and after light adaptation. RBC recordings were kept short (typically 2-5 $\mathrm{min}$ ) to minimize washout effects. All amacrine cells could be targeted particularly deep in the slice $(\sim 40-50 \mu \mathrm{m})$ and provided stable long lasting recordings ( $30 \mathrm{~min})$. To maximize the overlap in RBC sampling, we targeted pairs of A17 amacrine cells whose cell bodies were separated by less than $80 \mu \mathrm{m}$. 


\section{Assessment of $A 17 \rightarrow R B C$ connectivity}

To target A17 cells in whole mount mouse retina, the BAC lgfbp2 Gensat transgenic line (www.gensat. org) was reconstituted from cryo-frozen sperm (FVB background, stock\# 030560-UCD, www.mmrrc. org) using in vitro fertilization of Cd1/C57 hybrid eggs; mice were then bred into a C57/BL6 background. We confirmed morphologically that amacrine cells labeled in this line were A17 amacrines (Siegert et al., 2009) (Figure 4-figure supplement 1). GFP-positive A17 amacrine cells were targeted in 3- to 6-week-old mice. A17 cells were injected using sharp electrodes (tip resistance 150 M $\Omega$ ) with $2 \%$ Lucifer yellow (in $200 \mathrm{mM} \mathrm{KCl}$ ) prior to fixation with $4 \%$ paraformaldehyde in $0.1 \mathrm{M}$ phosphate buffered saline (PBS) for $20 \mathrm{~min}$. The retinas were rinsed with PBS and incubated for $72 \mathrm{hr}$ with rabbit polyclonal lucifer yellow (1:500, Invitrogen, Carlsbad, CA) and mouse monoclonal PKC (1:500, Sigma) antibodies in PBS with $0.5 \%$ Triton and $5 \%$ donkey serum. Retinas were incubated with secondary antibodies (antirabbit Alexa Fluor conjugate, Invitrogen and anti-mouse DyLight conjugate, Jackson ImmunoResearch, West Grove, PA) for 12 hr in PBS. Retinas were then mounted in Vectashield (Vector labs, Burlington, CA).

Images were acquired with an Olympus FV1000 microscope using a 1.35 NA 60x oil objective, at a voxel size of $0.102 \times 0.102 \times 0.3 \mu \mathrm{m}$ or $0.204 \times 0.204 \times 0.3 \mu \mathrm{m}$. Raw image stacks were processed with MetaMorph (Universal Imaging) and Amira (Mercury Computer Systems). To identify sites of apposition between PKC positive RBCs and A17 amacrine cell varicosities, pixel overlap was assessed upon rotation of the image volumes in 3D using Amira. Synaptic contact was defined when the fluorescent signals overlapped by $>1$ pixel at all angles of the $3 \mathrm{D}$ rotation. To determine the percentage of RBCs in the field of view that contacted the A17 cell, individual RBCs were digitally isolated and reconstructed using the 'label-field' function of Amira. To generate connectivity maps of the A17s, RBCs axonal locations were assessed in concentric rings spaced $20 \mu \mathrm{m}$ apart, centered on the soma of the injected A17. A17 dendrites traverse $\sim 40 \mu \mathrm{m}$ of the inner plexiform before reaching sublaminas 4 and 5 (where they make the majority of their synaptic contacts with RBCs); therefore, the most central concentric ring corresponds to dendritic distances between 40 and $60 \mu \mathrm{m}$, the second ring corresponds to dendritic distances between 60 and $80 \mu \mathrm{m}$ and so on.

The $A 17 \rightarrow R B C$ connectivity patterns derived from whole mount preparations were used to derive the slope factor that relates the RB's CSS to correlations measured in highly overlapping A17s (Equation 1 and Figure 4G). To account for the effects of slicing in our calculations, we divided the number of contacted RBCs within a given ring by two (except for the first ring). Using these values and Equation 1, we estimate the slope (purple line in Figure $4 G$ ) to be $0.64 \pm 0.03$ for highly overlapping A17s recorded in the slice preparation ( $0.67 \pm 0.03$ for whole mount).

\section{Electron microscropy}

A previously published data set was analyzed (Briggman et al., 2011) (retina k0563). Voxel dimensions were $12 \times 12 \times 25 \mathrm{~nm}^{3}$. Segmentation of identified RBCs, Alls, and A17s were performed using ITKSNAP (Yushkevich et al., 2006) (www.itksnap.org) and rendered in Matlab.

\section{Acknowledgements}

We thank Sid Kuo, Gautam Awatramani, Gabe Murphy for helpful comments on earlier versions of the paper, Felice Dunn for assistance with preliminary data collection and Mike Ahlquist, Mark Cafaro, Shellee Cunnington, and Paul Newman for outstanding technical assistance. Support provided by HHMI (FR), NIH Extramural Funding (EY11850 to FR, EY10699 to ROW) and the NINDS Intramural Research Program (KLB).

\section{Additional information}

Funding

\begin{tabular}{lll} 
Funder & Grant reference number & Author \\
\hline National Eye Institute & EY11850 & Fred Rieke \\
\hline Howard Hughes Medical Institute & & Fred Rieke \\
\hline National Eye Institute & 10699 & Rachel O Wong \\
\hline
\end{tabular}

The funders had no role in study design, data collection and interpretation, or the decision to submit the work for publication. 
Author contributions

WNG, MH, Conception and design, Acquisition of data, Analysis and interpretation of data, Drafting or revising the article; KLB, Acquisition of data, Analysis and interpretation of data, Drafting or revising the article; ROW, FR, Conception and design, Analysis and interpretation of data, Drafting or revising the article

Ethics

Animal experimentation: This work was performed in strict accordance with the recommendations in the Guide for the Care and Use of Laboratory Animals of the National Institutes of Health. All procedures followed protocols approved by the Institutional Animal Care and Use Committee (protocol 3030-01) of the University of Washington.

\section{References}

Ala-Laurila P, Greschner M, Chichilnisky EJ, Rieke F. 2011. Cone photoreceptor contributions to noise and correlations in the retinal output. Nature Neuroscience 14:1309-1316. doi: 10.1038/nn.2927.

Allen C, Stevens CF. 1994. An evaluation of causes for unreliability of synaptic transmission. Proceedings of the National Academy of Sciences of USA 91:10380-10383. doi: 10.1073/pnas.91.22.10380.

Arnett D, Spraker TE. 1981. Cross-correlation analysis of the maintained discharge of rabbit retinal ganglion cells. The Journal of Physiology 317:29-47.

Averbeck BB, Latham PE, Pouget A. 2006. Neural correlations, population coding and computation. Nature Reviews Neuroscience 7:358-366. doi: 10.1038/nrn1888.

Berntson A, Smith RG, Taylor WR. 2004. Transmission of single photon signals through a binary synapse in the mammalian retina. Visual Neuroscience 21:693-702.

Bloomfield SA, Dacheux RF. 2001. Rod vision: pathways and processing in the mammalian retina. Progress in Retinal and Eye Research 20:351-384. doi: 10.1016/S1350-9462(00)00031-8.

Briggman KL, Helmstaedter M, Denk W. 2011. Wiring specificity in the direction-selectivity circuit of the retina. Nature 471:183-188. doi: 10.1038/nature09818.

Brivanlou IH, Warland DK, Meister M. 1998. Mechanisms of concerted firing among retinal ganglion cells. Neuron 20:527-539. doi: 10.1016/S0896-6273(00)80992-7.

Cafaro J, Rieke F. 2010. Noise correlations improve response fidelity and stimulus encoding. Nature 468 : 964-967. doi: 10.1038/nature09570.

Deans MR, Gibson JR, Sellitto C, Conners BW, Paul DL. 2001. Synchronous activity of inhibitory networks in neocortex requires electrical synapses containing connexin36. Neuron 31:477-485. doi: 10.1016/S0896-6273 (01)00373-7.

Deans MR, Volgyi B, Goodenough DA, Bloomfield SA, Paul DL. 2002. Connexin36 is essential for transmission of rod-mediated visual signals in the mammalian retina. Neuron 36:703-712. doi: 10.1016/S0896-6273 (02)01046-2.

Del Castillo J, Katz B. 1954. Quantal components of the end-plate potential. The Journal of Physiology 124:560-573.

Deneve S, Latham PE, Pouget A. 2001. Efficient computation and cue integration with noisy population codes. Nature Neuroscience 4:826-831. doi: 10.1038/90541.

Denk W, Briggman KL, Helmstaedter M. 2012. Structural neurobiology: missing link to a mechanistic understanding of neural computation. Nature Reviews Neuroscience 13:351-358. doi: 10.1038/nrn3169.

Dunn FA, Doan T, Sampath AP, Rieke F. 2006. Controlling the gain of rod-mediated signals in the Mammalian retina. The Journal of Neuroscience 26:3959-3970. doi: 10.1523/JNEUROSCI.5148-05.2006.

Dunn FA, Rieke F. 2008. Single-photon absorptions evoke synaptic depression in the retina to extend the operational range of rod vision. Neuron 57:894-904. doi: 10.1016/j.neuron.2008.01.031.

Ellias SA, Stevens JK. 1980. The dendritic varicosity: a mechanism for electrically isolating the dendrites of cat retinal amacrine cells? Brain Research 196:365-372. doi: 10.1016/0006-8993(80)90401-1.

Euler T, Hausselt SE, Margolis DJ, Breuninger T, Castell X, Detwiler PB, Denk W. 2009. Eyecup scope-optical recordings of light stimulus-evoked fluorescence signals in the retina. Pflugers Archiv 457:1393-1414. doi: 10.1007/s00424-008-0603-5.

Field GD, Rieke F. 2002. Nonlinear signal transfer from mouse rods to bipolar cells and implications for visual sensitivity. Neuron 34:773-785. doi: 10.1016/S0896-6273(02)00700-6.

Field GD, Sampath AP, Rieke F. 2005. Retinal processing near absolute threshold: from behavior to mechanism. Annual Review of Physiology 67:491-514. doi: 10.1146/annurev.physiol.67.031103.151256.

Fischer BJ, Christianson GB, Pena JL. 2008. Cross-correlation in the auditory coincidence detectors of owls. The Journal of Neuroscience 28:8107-8115. doi: 10.1523/JNEUROSCI.1969-08.2008.

Grimes WN, Schwartz GW, Rieke F. 2014. The synaptic and circuit mechanisms underlying a change in spatial encoding in the retina. Neuron 82:460-473. doi: 10.1016/j.neuron.2014.02.037.

Grimes WN, Zhang J, Graydon CW, Kachar B, Diamond JS. 2010. Retinal parallel processors: more than 100 independent microcircuits operate within a single interneuron. Neuron 65:873-885. doi: 10.1016/j.neuron. 2010.02.028. 
Haverkamp S, Wassle H. 2000. Immunocytochemical analysis of the mouse retina. The Journal of Comparative Neurology 424:1-23. doi: 10.1002/1096-9861(20000814)424:13.0.CO;2-V.

Hecht S, Shlaer S, Pirenne MH. 1942. Energy, quanta, and vision. The Journal of General Physiology 25:819-840. doi: 10.1085/jgp.25.6.819.

Jarsky T, Cembrowski M, Logan SM, Kath WL, Riecke H, Demb JB, Singer JH. 2011. A synaptic mechanism for retinal adaptation to luminance and contrast. The Journal of Neuroscience 31:11003-11015. doi: 10.1523/ JNEUROSCI.2631-11.2011.

Kazama H, Wilson Rl. 2009. Origins of correlated activity in an olfactory circuit. Nature Neuroscience 12 : 1136-1144. doi: 10.1038/nn.2376.

Ke JB, Wang YV, Borghuis BG, Cembrowski MS, Riecke H, Kath WL, Demb JB, Singer JH. 2014. Adaptation to background light enables contrast coding at rod bipolar cell synapses. Neuron 81:388-401. doi: 10.1016/j. neuron.2013.10.054.

Kolb H. 1970. Organization of the outer plexiform layer of the primate retina: electron microscopy of Golgiimpregnated cells. Philosophical Transactions of the Royal Society of London 258:261-283. doi: 10.1098/ rstb.1970.0036.

Kolb H, Famiglietti EV. 1974. Rod and cone pathways in the inner plexiform layer of cat retina. Science 186: 47-49. doi: 10.1126/science.186.4158.47.

Manookin MB, Beaudoin DL, Ernst ZR, Flagel LJ, Demb JB. 2008. Disinhibition combines with excitation to extend the operating range of the OFF visual pathway in daylight. The Journal of Neuroscience 28:4136-4150. doi: 10.1523/JNEUROSCI.4274-07.2008.

Mastronarde DN. 1983. Correlated firing of cat retinal ganglion cells. I. Spontaneously active inputs to X- and Y-cells. Journal of Neurophysiology 49:303-324.

Mehta B, Ke JB, Zhang L, Baden AD, Markowitz AL, Nayak S, Briggman KL, Zenisek D, Singer JH. 2014. Global $\mathrm{Ca} 2+$ signaling drives ribbon-independent synaptic transmission at rod bipolar cell synapses. Journal of Neuroscience 34:6233-6244. doi: 10.1523/JNEUROSCI.5324-13.2014.

Munch TA, da Silveira RA, Siegert S, Viney TJ, Awatramani GB, Roska B. 2009. Approach sensitivity in the retina processed by a multifunctional neural circuit. Nature Neuroscience 12:1308-1316. doi: 10.1038/nn.2389.

Murphy GJ, Rieke F. 2006. Network variability limits stimulus-evoked spike timing precision in retinal ganglion cells. Neuron 52:511-524. doi: 10.1016/j.neuron.2006.09.014.

Murphy GJ, Rieke F. 2008. Signals and noise in an inhibitory interneuron diverge to control activity in nearby retinal ganglion cells. Nature Neuroscience 11:318-326. doi: 10.1038/nn2045.

Naarendorp F, Esdaille TM, Banden SM, Andrews-Labenski J, Gross OP, Pugh EN Jnr. 2010. Dark light, rod saturation, and the absolute and incremental sensitivity of mouse cone vision. The Journal of Neuroscience 30:12495-12507. doi: 10.1523/JNEUROSCI.2186-10.2010.

Nelson R, Kolb H. 1984. Amacrine cells in scotopic vision. Ophthalmic Research 16:21-26. doi: 10.1159/000265288.

Nelson R, Kolb H. 1985. A17: a broad-field amacrine cell in the rod system of the cat retina. Journal of Neurophysiology 54:592-614.

Oesch NW, Diamond JS. 2011. Ribbon synapses compute temporal contrast and encode luminance in retinal rod bipolar cells. Nature Neuroscience 14:1555-1561. doi: 10.1038/nn.2945.

Olsen SR, Bhandawat V, Wilson RI. 2007. Excitatory interactions between olfactory processing channels in the Drosophila antennal lobe. Neuron 54:89-103. doi: 10.1016/j.neuron.2007.03.010.

Protti DA, Llano I. 1998. Calcium currents and calcium signaling in rod bipolar cells of rat retinal slices. The Journal of Neuroscience 18:3715-3724.

Rieke F, Baylor DA. 1998. Origin of reproducibility in the responses of retinal rods to single photons. Biophysical Journal 75:1836-1857. doi: 10.1016/S0006-3495(98)77625-8.

Rosenbaum R, Rubin JE, Doiron B. 2013. Short-term synaptic depression and stochastic vesicle dynamics reduce and shape neuronal correlations. Journal of Neurophysiology 109:475-484. doi: 10.1152/jn.00733.2012.

Rosenbaum R, Zimnik A, Zheng F, Turner RS, Alzheimer C, Doiron B, Rubin JE. 2014. Axonal and synaptic failure suppress the transfer of firing rate oscillations, synchrony and information during high frequency deep brain stimulation. Neurobiology of Disease 62:86-99. doi: 10.1016/j.nbd.2013.09.006.

Salinas E, Sejnowski TJ. 2000. Impact of correlated synaptic input on output firing rate and variability in simple neuronal models. The Journal of Neuroscience 20:6193-6209.

Sampath AP, Rieke F. 2004. Selective transmission of single photon responses by saturation at the rod-to-rod bipolar synapse. Neuron 41:431-443. doi: 10.1016/S0896-6273(04)00005-4.

Schnell B, Joesch M, Forstner F, Raghu SV, Otsuna H, Ito K, Borst A, Reiff DF. 2010. Processing of horizontal optic flow in three visual interneurons of the Drosophila brain. Journal of Neurophysiology 103:1646-1657. doi: 10.1152/jn.00950.2009.

Siegert S, Scherf BG, Del Punta K, Didkovsky N, Heintz N, Roska B. 2009. Genetic address book for retinal cell types. Nature Neuroscience 12:1197-1204. doi: 10.1038/nn.2370.

Singer JH, Diamond JS. 2006. Vesicle depletion and synaptic depression at a mammalian ribbon synapse. Journal of Neurophysiology 95:3191-3198. doi: 10.1152/jn.01309.2005.

Slaughter MM, Miller RF. 1981. 2-amino-4-phosphonobutyric acid: a new pharmacological tool for retina research. Science 211:182-185. doi: 10.1126/science.6255566.

Sterling P, Lampson LA. 1986. Molecular specificity of defined types of amacrine synapse in cat retina. The Journal of Neuroscience 6:1314-1324.

Tong G, Jahr CE. 1994. Multivesicular release from excitatory synapses of cultured hippocampal neurons. Neuron 12:51-59. doi: 10.1016/0896-6273(94)90151-1. 
Trexler EB, Li W, Massey SC. 2005. Simultaneous contribution of two rod pathways to All amacrine and cone bipolar cell light responses. Journal of Neurophysiology 93:1476-1485. doi: 10.1152/jn.00597.2004.

Trong PK, Rieke F. 2008. Origin of correlated activity between parasol retinal ganglion cells. Nature Neuroscience 11:1343-1351. doi: 10.1038/nn.2199.

Tsukamoto Y, Morigiwa K, Ueda M, Sterling P. 2001. Microcircuits for night vision in mouse retina. The Journal of Neuroscience 21:8616-8623.

Tsukamoto Y, Omi N. 2013. Functional allocation of synaptic contacts in microcircuits from rods via rod bipolar to All amacrine cells in the mouse retina. The Journal of Comparative Neurology 521:3541-3555. doi: 10.1002/cne.23370.

van Rossum MC, Smith RG. 1998. Noise removal at the rod synapse of mammalian retina. Visual Neuroscience 15:809-821. doi: 10.1017/S0952523898155037.

Volgyi B, Pan F, Paul DL, Wang JT, Huberman AD, Bloomfield SA. 2013. Gap junctions are essential for generating the correlated spike activity of neighboring retinal ganglion cells. PLOS ONE 8:e69426. doi: 10.1371/journal. pone.0069426.

Xin D, Bloomfield SA. 1999. Comparison of the responses of All amacrine cells in the dark- and light-adapted rabbit retina. Visual Neuroscience 16:653-665. doi: 10.1017/S0952523899164058.

Yushkevich PA, Piven J, Hazlett HC, Smith RG, Ho S, Gee JC, Gerig G. 2006. User-guided 3D active contour segmentation of anatomical structures: significantly improved efficiency and reliability. Neuroimage 31 : 1116-1128. doi: 10.1016/j. neuroimage.2006.01.015.

Zhang J, Li W, Trexler EB, Massey SC. 2002. Confocal analysis of reciprocal feedback at rod bipolar terminals in the rabbit retina. The Journal of Neuroscience 22:10871-10882. 